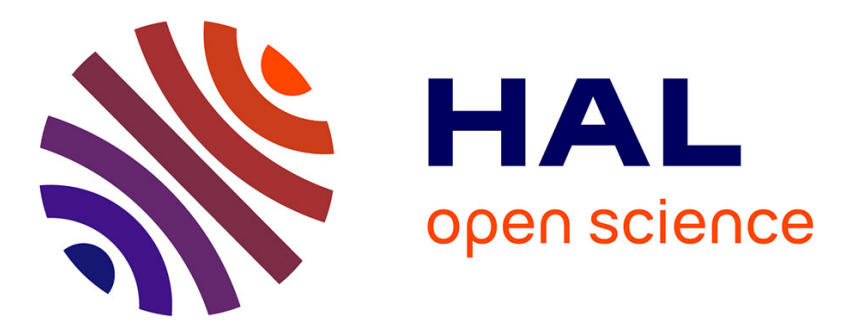

\title{
Hybridization of Mixed High-Order Methods on General Meshes and Application to the Stokes Equations
}

\author{
Joubine Aghili, Sébastien Boyaval, Daniele Di Pietro
}

\section{To cite this version:}

Joubine Aghili, Sébastien Boyaval, Daniele Di Pietro. Hybridization of Mixed High-Order Methods on General Meshes and Application to the Stokes Equations. Computational Methods in Applied Mathematics, 2015, 10.1515/cmam-2015-0004 . hal-01009723v2

\section{HAL Id: hal-01009723 \\ https://hal.science/hal-01009723v2}

Submitted on 22 Feb 2015

HAL is a multi-disciplinary open access archive for the deposit and dissemination of scientific research documents, whether they are published or not. The documents may come from teaching and research institutions in France or abroad, or from public or private research centers.
L'archive ouverte pluridisciplinaire HAL, est destinée au dépôt et à la diffusion de documents scientifiques de niveau recherche, publiés ou non, émanant des établissements d'enseignement et de recherche français ou étrangers, des laboratoires publics ou privés. 


\title{
Hybridization of mixed high-order methods on general meshes and application to the Stokes equations
}

\author{
Joubine Aghili*1, Sébastien Boyaval ${ }^{\dagger 2}$, and Daniele A. Di Pietro ${ }^{\ddagger 1}$ \\ ${ }^{1}$ Université de Montpellier, I3M, 34057 Montpellier CEDEX 5, France \\ ${ }^{2}$ Laboratoire d'hydraulique Saint-Venant (Ecole des Ponts ParisTech - EDF R\&D - CEREMA), \\ Université Paris Est and INRIA Rocquencourt (MATHERIALS)
}

February 16, 2015

\begin{abstract}
This paper presents two novel contributions on the recently introduced Mixed HighOrder (MHO) methods [19]. We first address the hybridization of the MHO method for a scalar diffusion problem and obtain the corresponding primal formulation. Based on the hybridized MHO method, we then design a novel, arbitrary order method for the Stokes problem on general meshes. A full convergence analysis is carried out showing that, when independent polynomials of degree $k$ are used as unknowns (at elements for the pressure and at faces for each velocity component), the energy-norm of the velocity and the $L^{2}$-norm of the pressure converge with order $(k+1)$, while the $L^{2}$-norm of the velocity (super-)converges with order $(k+2)$. The latter property is not shared by other methods based on a similar choice of unknowns. The theoretical results are numerically validated in two space dimensions on both standard and polygonal meshes.
\end{abstract}

Keywords Stokes, general meshes, mixed high-order methods, hybridization

\section{Introduction}

Approximation methods on general polygonal or polyhedral meshes are an active field of research. The interest in handling general meshes can be prompted, e.g., by the desire to adapt the element shape to the qualitative features of the solution (elongated hexahedral elements in boundary layers combined with tetrahedra in the interior of the domain) and by nonconforming or agglomerative mesh adaptation, cf., e.g., Bassi et al. [4] . A wide range of low-order numerical methods have been proposed over the last years that handle general polygonal or polyhedral discretizations; cf., e.g., [23, 24] for a review. More recently, higherorder methods have also been considered. High-order MFD schemes have been studied by Beirão da Veiga, Lipnikov, and Manzini [9]; see also [34] for recent developments. We cite here also the Virtual Element Method (VEM) introduced by Beirão da Veiga, Brezzi, and Marini [6] (cf. also [5]). In [19] and [21], different approaches are considered inspired by classical mixed

\footnotetext{
*joubine.aghili@univ-montp2.fr, corresponding author

${ }^{\dagger}$ sebastien.boyaval@enpc.fr

${ }^{\ddagger}$ daniele.di-pietro@univ-montp2.fr
} 
(Raviart-Thomas) and non-conforming (Crouzeix-Raviart) finite elements, leading to socalled Mixed High-Order (MHO) and Hybrid High-Order (HHO) methods, respectively. We refer here also to [20] for an application to linear elasticity and to the work of Vohralik and Wohlmuth [37] for another perspective on mixed methods on general meshes. Very recently, $\boldsymbol{H}(\operatorname{div} ; \Omega)$-conforming VEM have been proposed [7] which are suitable for devising mixed discretizations on general meshes, and which seem to share some features with MHO.

For a scalar diffusion problem, Hybrid High-Order methods (and, as we will see in Section 3.3, MHO methods after hybridization) use as intermediate unknowns polynomials of degree $k$ at elements, and lead to a problem where the only globally coupled unknowns are polynomials of degree $k$ at mesh faces. Also the Hybridizable Discontinuous Galerkin (HDG) methods of Cockburn, Gopalakrishnan, and Lazarov [15] can be written with a similar choice of unknowns by locally eliminating the flux variable, thereby obtaining, after hybridization, a global problem with the same number of unknowns and stencil, cf. also [26]. In this case, convergence with order $(k+1)$ is observed for the $L^{2}$-norms of both the flux and of the potential on general meshes (cf. the analysis in Castillo, Cockburn, Perugia, and Schötzau [12], which extends to HDG). On the other hand, for an analogous choice of unknowns, MHO/HHO methods converge with order $(k+1)$ in the (discrete) energy norm and $(k+2)$ in the $L^{2}$ potential norm. This means that superconvergence (or, more appropriately, supercloseness) of the potential holds. In the context of HDG methods, similar convergence results can be obtained using as element unknowns polynomials of degree $(k+1)$ instead of $k$, and tweaking the stabilization as proposed by Lehrenfeld [33, Remark 1.2.4]. It is useful to stress that the announced orders of convergence for $\mathrm{MHO} / \mathrm{HHO}$ are obtained for general meshes and arbitrary polynomial degree $k$. This is achieved by (i) introducing a reconstruction of the flux for MHO (cf. (21) below) and of the gradient for HHO (cf. (46) below) of order $(k+1)$ obtained by solving a local Neumann problem inside each element and (ii) designing the penalty term in a careful way so as to preserve the order of the reconstruction. An in-depth study of the relation between $\mathrm{MHO} / \mathrm{HHO}$ and HDG methods can be found in [14].

This paper presents two novel contributions:

(i) we hybridize the MHO method of [19] in the spirit of [2] and clarify its link with the HHO method of [21]. This section contains an interesting novel result bridging MHO and HHO methods, and clarifying the analogies and differences (essentially related to the choice of the stabilization term);

(ii) based on the hybrized MHO method, we design and analyze a novel arbitrary-order, inf-sup stable method for the Stokes equations. A full convergence analysis is performed including both energy- and $L^{2}$-norm estimates.

After static condensation of the element unknowns for the velocity, the unknowns for the Stokes problem are vector-valued polynomials of degree $k \geqslant 0$ at faces for the velocity and polynomials of degree $k$ at elements for the pressure. The choice of polynomials of degree $k$ for the pressure justifies itself observing that the method relies in fact on a polynomial velocity reconstruction of degree $(k+1)$. The key features of the method can be summarized as follows:

(i) it supports general polyhedral meshes and arbitrary approximation order (including $k=0$, which is not usually the case for discontinuous Galerkin methods); 
(ii) when polynomials of degree $k$ are used as velocity unknowns, the order of convergence is $(k+1)$ for both the energy-norm of the velocity and the $L^{2}$-norm of the pressure and $(k+2)$ for the $L^{2}$-norm of the velocity;

(iii) it has reduced computational cost. After locally condensing the element unknowns for the velocity, the lowest-order version of the method in dimension $d$ only requires $d$ unknowns per face for the velocity and one unknown per element for the pressure.

It is also worth mentioning that, for $k \geqslant 1$, partial static condensation can also be applied for the pressure variable, leaving only one pressure unknown per element globally coupled (this unknown represents the average value of the pressure on the element).

To conclude, we briefly review some methods for the Stokes problem based on hybrid spaces for the velocity. A HDG method for Stokes flows is proposed by Nguyen, Peraire, and Cockburn [35]. In this case, polynomials of degree $k$ are used for the flux, velocity, and pressure variables, and an order of convergence of $(k+1)$ is experimentally obtained for the $L^{2}$-norm of the error in each variable (we recall that, in our case, the $L^{2}$-norm of the error on the velocity converges with order $(k+2)$, cf. Theorem 9$)$. Similar considerations apply for the methods presented in [16]. In [32], Labeur and Wells present a HDG methods where the velocity unknowns are polynomials of degree $k$ at elements and faces. Also in this case, the $L^{2}$-errors on both the velocity and the pressure approximations converge with order $(k+1)$. In [27], on the other hand, Egger and Waluga consider a method where polynomials of degree $k$ and $(k-1)$ are used for the velocity and the pressure, respectively, and a $h p$-convergence analysis is carried out. In this case, both the norm of the velocity gradient and the $L^{2}$-norm of the pressure converge with order $k$. Other work on the discretization of the Stokes equations that deserves being mentioned here include the $h p$-discontinuous Galerkin method of Toselli [36], the domain decomposition method of Girault, Rivière, and Wheeler [29], and the hybridized globally divergence free LDG method of Carrero, Cockburn, and Schötzau [11].

The paper is structured as follows. In Section 2, we briefly recall the main assumptions on the mesh in the spirit of [18] as well as some basic results on broken functional spaces used in the analysis. In Section 3, we carry out hybridization for the MHO method of [19] and show that the resulting hybridized MHO method differs from the HHO method of [21] only by the choice of the stabilization term. In Section 4, we derive a novel method for the Stokes problem, perform a full convergence analysis in the energy- and $L^{2}$-norms, and provide numerical validation of the estimates on both standard and general polygonal meshes. Finally, implementation aspects are thoroughly discussed in Section 5.

\section{Setting}

In this section we briefly recall the notion of admissible mesh sequences introduced in $[18$, Chapter 1] as well as some basic results for broken functional spaces.

\subsection{Admissible mesh sequences}

Throughout the rest of the paper, $\Omega$ denotes an open, connected, bounded polygonal or polyhedral domain in $\mathbb{R}^{d}, d \geqslant 1$. For any open, connected subset $X \subset \bar{\Omega}$ with non-zero 
Lebesgue measure, the standard inner product and norm of the Lebesgue space $L^{2}(X)$ are denoted by $(\cdot, \cdot)_{X}$ and $\|\cdot\|_{X}$, respectively, with the convention that the index is omitted if $X=\Omega$.

Denoting by $\mathcal{H} \subset \mathbb{R}_{*}^{+}$a countable set of meshsizes having 0 as its unique accumulation point, we consider mesh sequences $\left(\mathcal{T}_{h}\right)_{h \in \mathcal{H}}$ where, for all $h \in \mathcal{H}, \mathcal{T}_{h}=\{T\}$ is a finite collection of nonempty disjoint open polyhedra $T$ (called elements or cells) such that $\bar{\Omega}=\bigcup_{T \in \mathcal{T}_{h}} \bar{T}$ and $h=\max _{T \in \mathcal{T}_{h}} h_{T}\left(h_{T}\right.$ stands for the diameter of $\left.T\right)$.

A hyperplanar closed connected subset $F$ of $\bar{\Omega}$ is called a face if it has positive $(d-1)$ dimensional measure and (i) either there exist $T_{1}, T_{2} \in \mathcal{T}_{h}$ such that $F \subset \partial T_{1} \cap \partial T_{2}$ (and $F$ is an interface) or (ii) there exists $T \in \mathcal{T}_{h}$ such that $F \subset \partial T \cap \partial \Omega$ (and $F$ is a boundary face). The set of interfaces is denoted by $\mathcal{F}_{h}^{\mathrm{i}}$, the set of boundary faces by $\mathcal{F}_{h}^{\mathrm{b}}$, and we let $\mathcal{F}_{h}:=\mathcal{F}_{h}^{\mathrm{i}} \cup \mathcal{F}_{h}^{\mathrm{b}}$. The diameter of a face $F \in \mathcal{F}_{h}$ is denoted by $h_{F}$

For all $T \in \mathcal{T}_{h}$, we let $\mathcal{F}_{T}:=\left\{F \in \mathcal{F}_{h} \mid F \subset \partial T\right\}$ denote the set of faces lying on the boundary of $T$. Symmetrically, for all $F \in \mathcal{F}_{h}, \mathcal{T}_{F}:=\left\{T \in \mathcal{T}_{h} \mid F \subset \partial T\right\}$ is the set of the one (if $F$ is a boundary face) or two (if $F$ is an interface) elements sharing $F$.

For all $F \in \mathcal{F}_{T}$, we denote by $\boldsymbol{n}_{T F}$ the normal to $F$ pointing out of $T$. For every interface $F \subset \partial T_{1} \cap \partial T_{2}$, we adopt the following convention: an orientation is fixed once and for all by means of a unit normal vector $\boldsymbol{n}_{F}$, and the elements $T_{1}$ and $T_{2}$ are numbered so that $\boldsymbol{n}_{F}:=\boldsymbol{n}_{T_{1} F}$.

We assume throughout the rest of this work that the mesh sequence $\left(\mathcal{T}_{h}\right)_{\mathcal{H}}$ is admissible in the sense of $\left[18\right.$, Chapter 1], i.e., for all $h \in \mathcal{H}, \mathcal{T}_{h}$ admits a matching simplicial submesh $\mathfrak{T}_{h}$ and the following properties hold for all $h \in \mathcal{H}$ with mesh regularity parameter $\varrho>0$ independent of $h$ : (i) for all simplex $S \in \mathfrak{T}_{h}$ of diameter $h_{S}$ and inradius $r_{S}, \varrho h_{S} \leqslant r_{S}$ and (ii) for all $T \in \mathcal{T}_{h}$, and all $S \in \mathfrak{T}_{T}:=\left\{S \in \mathfrak{T}_{h} \mid S \subset T\right\}, \varrho h_{T} \leqslant h_{S}$. For an admissible mesh sequence, it is known from [18, Lemma 1.41] that the number of faces of one element can be bounded uniformly in $h$, i.e., it holds that

$$
\forall h \in \mathcal{H}, \quad \max _{T \in \mathcal{T}_{h}}\left\{\mathfrak{N}_{T}:=\operatorname{card}\left(\mathcal{F}_{T}\right)\right\} \leqslant \mathfrak{N}_{\partial},
$$

for an integer $(d+1) \leqslant \mathfrak{N}_{\partial}<+\infty$ independent of $h$. Furthermore, for all $h \in \mathcal{H}, T \in \mathcal{T}_{h}$ and $F \in \mathcal{F}_{T}, h_{F}$ is comparable to $h_{T}$ in the following sense (cf. [18, Lemma 1.42]): $\rho^{2} h_{T} \leqslant h_{F} \leqslant$ $h_{T}$.

\subsection{Basic results on broken functional spaces}

We next state some basic results that hold for broken functional spaces on admissible mesh sequences $\left(\mathcal{T}_{h}\right)_{h \in \mathcal{H}}$.

Let an integer $k \geqslant 0$ be fixed. For all $T \in \mathcal{T}_{h}$ and all $v \in \mathbb{P}_{d}^{k}(T)\left(\mathbb{P}_{d}^{k}(T)\right.$ is spanned by the restriction to $T$ of $d$-variate polynomial functions of total degree $\leqslant k$ ), the following trace and inverse inequalities hold:

$$
\begin{aligned}
\|v\|_{F} & \leqslant C_{\text {tr }} h_{F}^{-1 / 2}\|v\|_{T} \quad \forall F \in \mathcal{F}_{T}, \\
\|\nabla v\|_{T} & \leqslant C_{\text {inv }} h_{T}^{-1}\|v\|_{T},
\end{aligned}
$$


with real numbers $C_{\text {tr }}$ and $C_{\text {inv }}$ that are independent of $h \in \mathcal{H}$, cf. [18, Lemmata 1.44 and 1.46].

Using [18, Lemma 1.40] together with the results of [25], one can prove the existence of a real number $C_{\text {app }}$ independent of $h$ such that, for all $T \in \mathcal{T}_{h}$, the $L^{2}$-orthogonal projector $\pi_{T}^{k}$ on $\mathbb{P}_{d}^{k}(T)$ satisfies: For all $s \in\{0, \ldots, k+1\}$, and all $v \in H^{s}(T)$,

$$
\left|v-\pi_{T}^{k} v\right|_{H^{m}(T)} \leqslant C_{\mathrm{app}} h_{T}^{s-m}|v|_{H^{s}(T)} \quad \forall m \in\{0, \ldots, s\} .
$$

This will be our reference convergence rate for the approximation of the solutions to (isotropic) diffusion problems. In what follows, we also need the $L^{2}$-orthogonal operator $\pi_{h}^{k}$ on the broken polynomial space

$$
\mathbb{P}_{d}^{k}\left(\mathcal{T}_{h}\right):=\left\{v \in L^{2}(\Omega) \mid v_{\mid T} \in \mathbb{P}_{d}^{k}(T) \quad \forall T \in \mathcal{T}_{h}\right\}
$$

Clearly, for all $v \in L^{2}(\Omega)$, and all $T \in \mathcal{T}_{h}$, it holds that $\pi_{T}^{k} v_{\mid T}=\left(\pi_{h}^{k} v\right)_{\mid T}$, and optimal approximation properties for $\pi_{h}^{k}$ follow from (4). Finally, we also introduce the notation $H^{l}\left(\mathcal{T}_{h}\right):=\left\{v \in L^{2}(\Omega) \mid v_{\mid T} \in H^{l}(T)\right\}$ for broken Sobolev spaces.

For the sake of conciseness, in what follows we often abbreviate by $a \lesssim b$ the inequality $a \leqslant C b$ with generic constant $C>0$ independent of $h$ but possibly depending on the mesh regularity parameter $\varrho$ and on the polynomial degree $k$.

\section{A hybridized arbitrary-order discretization for diffusion terms}

As a preliminary step to design the discretization of viscous terms in the Stokes equations (cf. Section 4$)$, we consider here the Laplace equation $\left(f \in L^{2}(\Omega)\right.$ denotes the forcing term),

$$
-\triangle u=f \quad \text { in } \Omega, \quad u=0 \quad \text { on } \partial \Omega \text {. }
$$

Letting

$$
\boldsymbol{\Sigma}:=\boldsymbol{H}(\operatorname{div} ; \Omega), \quad U:=L^{2}(\Omega),
$$

the mixed variational formulation of problem (6) reads: Find $(s, u) \in \boldsymbol{\Sigma} \times U$ such that

$$
\begin{array}{rlrl}
(\boldsymbol{s}, \boldsymbol{t})+(u, \boldsymbol{\nabla} \cdot \boldsymbol{t}) & =0 & & \forall \boldsymbol{t} \in \boldsymbol{\Sigma}, \\
-(\boldsymbol{\nabla} \cdot \boldsymbol{s}, v) & =(f, v) & \forall v \in U .
\end{array}
$$

The unknowns $s$ and $u$ will be henceforth referred to as the flux and potential, respectively. At the continuous level, a primal formulation where the potential $u$ appears as the sole unknown can be obtained by eliminating the flux $s$. A discrete counterpart of this procedure (the so-called hybridization) is studied here for the MHO method of [19]. An important result of this section is that we establish a link between the resulting coercive problem and the Hybrid High-Order method of [21], which corresponds to a different (but equivalent in a sense that will be made precise) choice of the penalty term.

\subsection{A Mixed High-Order discretization of diffusion}

In this section, we recall the MHO discretization of diffusive terms from [19] as well as a few results that will be useful for the subsequent discussion. 


\subsubsection{Degrees of freedom}

Given any fixed integer $k \geqslant 0$, the generalized flux degrees of freedom (DOFs) for the mixed method are defined as

$$
\mathbb{T}_{T}^{k}:=\nabla \mathbb{P}_{d}^{k, 0}(T) \quad \forall T \in \mathcal{T}_{h}, \quad \mathbb{F}_{F}^{k}:=\mathbb{P}_{d-1}^{k}(F) \quad \forall F \in \mathcal{F}_{h},
$$

where for $l \geqslant 0, \mathbb{P}_{d}^{l, 0}(T)$ is spanned by scalar-valued polynomial functions of total degree $\leqslant l$ with zero average on $T$. Note that, in the lowest-order case $k=0, \mathbb{T}_{T}^{k}$ has dimension zero, which reflects the fact that cell DOFs are unnecessary. The local and global DOF spaces for the flux approximation in MHO are, respectively,

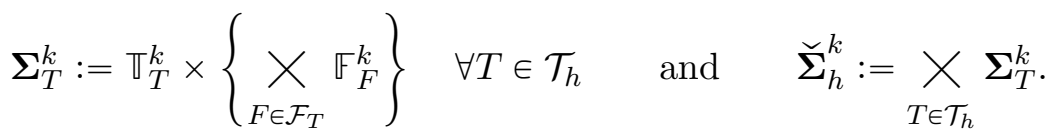

We also introduce the following patched version of $\breve{\Sigma}_{h}^{k}$ :

$$
\boldsymbol{\Sigma}_{h}^{k}:=\left\{\boldsymbol{\tau}_{h}=\left(\boldsymbol{\tau}_{T},\left(\tau_{T F}\right)_{F \in \mathcal{F}_{T}}\right)_{T \in \mathcal{T}_{h}} \in \check{\boldsymbol{\Sigma}}_{h}^{k} \mid \sum_{T \in \mathcal{T}_{F}} \tau_{T F}=0 \quad \forall F \in \mathcal{F}_{h}^{\mathrm{i}}\right\}
$$

The local space $\boldsymbol{\Sigma}_{T}^{k}$ is equipped with the following norm:

$$
\forall \boldsymbol{\tau} \in \boldsymbol{\Sigma}_{T}^{k}, \quad\|\boldsymbol{\tau}\|_{T}^{2}:=\left\|\boldsymbol{\tau}_{T}\right\|_{T}^{2}+\sum_{F \in \mathcal{F}_{T}} h_{F}\left\|\tau_{T F}\right\|_{F}^{2} .
$$

For all $T \in \mathcal{T}_{h}$, we denote by $R_{\Sigma, T}^{k}: \check{\Sigma}_{h}^{k} \rightarrow \Sigma_{T}^{k}$ the restriction operator which realizes the mapping between global and local flux DOFs, and we equip $\check{\Sigma}_{h}^{k}$ with the norm

$$
\forall \boldsymbol{\tau}_{h} \in \check{\Sigma}_{h}^{k}, \quad\left\|\boldsymbol{\tau}_{h}\right\|^{2}:=\sum_{T \in \mathcal{T}_{F}}\left\|R_{\Sigma, T}^{k} \boldsymbol{\tau}_{h}\right\|_{T}^{2} .
$$

Let, for a fixed $s>2, \boldsymbol{\Sigma}^{+}(T):=\left\{\boldsymbol{t} \in L^{s}(T)^{d} \mid \boldsymbol{\nabla} \cdot \boldsymbol{t} \in L^{2}(T)\right\}$. The regularity in $\boldsymbol{\Sigma}^{+}(T)$ is required to ensure that the $L^{2}$-orthogonal projector $\pi_{F}^{k}$ over $\mathbb{F}_{F}^{k}=\mathbb{P}_{d}^{k}(F)$ is well-defined for all $F \in \mathcal{F}_{T}$, cf. e.g., [28, Section 1.2.7]. We define the local interpolator $I_{\boldsymbol{\Sigma}, T}^{k}: \boldsymbol{\Sigma}^{+}(T) \rightarrow \boldsymbol{\Sigma}_{T}^{k}$ such that, for all $\boldsymbol{t} \in \boldsymbol{\Sigma}^{+}(T), I_{\boldsymbol{\Sigma}, T}^{k} \boldsymbol{t}=\left(\boldsymbol{\tau}_{T},\left(\tau_{T F}\right)_{F \in \mathcal{F}_{T}}\right)$ with

$$
\boldsymbol{\tau}_{T}=\varpi_{T}^{k} \boldsymbol{t}, \quad \tau_{T F}=\pi_{F}^{k}\left(\boldsymbol{t} \cdot \boldsymbol{n}_{T F}\right) \quad \forall F \in \mathcal{F}_{T},
$$

where $\varpi_{T}^{k}$ denotes the $L^{2}$-orthogonal projector on $\mathbb{T}_{T}^{k}$ (in fact, an elliptic projector on $\mathbb{P}_{d}^{k, 0}(T)$ ) such that

$$
\left(\varpi_{T}^{k} \boldsymbol{t}, \boldsymbol{w}\right)_{T}=(\boldsymbol{t}, \boldsymbol{w})_{T} \quad \forall \boldsymbol{w} \in \mathbb{T}_{T}^{k} .
$$

The global interpolator $I_{\boldsymbol{\Sigma}, h}^{k}: \boldsymbol{\Sigma}^{+} \rightarrow \check{\boldsymbol{\Sigma}}_{h}^{k}$ with $\boldsymbol{\Sigma}^{+}:=\left\{\boldsymbol{t} \in L^{2}(\Omega)^{d} \mid \boldsymbol{t}_{\mid T} \in \boldsymbol{\Sigma}^{+}(T), \forall T \in \mathcal{T}_{h}\right\}$ is such that, for all $\boldsymbol{t} \in \boldsymbol{\Sigma}^{+}$,

$$
R_{\Sigma, T}^{k} I_{\Sigma, h}^{k} \boldsymbol{t}=I_{\Sigma, T}^{k} \boldsymbol{t}_{\mid T} \quad \forall T \in \mathcal{T}_{h} .
$$

Remark 1 (Restriction of $I_{\boldsymbol{\Sigma}, h}^{k}$ to $\boldsymbol{\Sigma}^{+} \cap \boldsymbol{H}(\operatorname{div} ; \Omega)$ ). An important remark is that functions in $\boldsymbol{\Sigma}^{+} \cap \boldsymbol{H}(\operatorname{div} ; \Omega)$ are mapped by $I_{\boldsymbol{\Sigma}, h}^{k}$ to elements of the patched space $\boldsymbol{\Sigma}_{h}^{k}$, cf. (11). 
The local and global DOF spaces for the potential are given by, respectively,

$$
U_{T}^{k}:=\mathbb{P}_{d}^{k}(T) \quad \forall T \in \mathcal{T}_{h} \quad \text { and } \quad U_{h}^{k}:=\underset{T \in \mathcal{T}_{h}}{\chi} U_{T}^{k} .
$$

In the following, we identify when needed the space $U_{h}^{k}$ with the broken polynomial space $\mathbb{P}_{d}^{k}\left(\mathcal{T}_{h}\right)$ defined by (5). Both $U_{T}^{k}$ and $U_{h}^{k}$ are naturally endowed with the $L^{2}$-norm topology.

\subsubsection{Divergence and flux reconstructions}

Let $T \in \mathcal{T}_{h}$. We define the local discrete divergence operator $D_{T}^{k}: \Sigma_{T}^{k} \rightarrow \mathbb{P}_{d}^{k}(T)$ such that, for all $\boldsymbol{\tau}=\left(\boldsymbol{\tau}_{T},\left(\tau_{T F}\right)_{F \in \mathcal{F}_{T}}\right) \in \boldsymbol{\Sigma}_{T}^{k}$,

$$
\left(D_{T}^{k} \boldsymbol{\tau}, v\right)_{T}=-\left(\boldsymbol{\tau}_{T}, \boldsymbol{\nabla} v\right)_{T}+\sum_{F \in \mathcal{F}_{T}}\left(\tau_{T F}, v\right)_{F}, \quad \forall v \in \mathbb{P}_{d}^{k}(T) .
$$

The operator $D_{T}^{k}$ is designed so as to satisfy the following commuting diagram property:

$$
D_{T}^{k}\left(I_{\Sigma, T}^{k} \boldsymbol{t}\right)=\pi_{T}^{k}(\boldsymbol{\nabla} \cdot \boldsymbol{t}) \quad \forall \boldsymbol{t} \in \boldsymbol{\Sigma}^{+}(T) .
$$

Its global counterpart $D_{h}^{k}: \check{\Sigma}_{h}^{k} \rightarrow \mathbb{P}_{d}^{k}\left(\mathcal{T}_{h}\right)$ is such that, for all $\boldsymbol{\tau}_{h} \in \breve{\boldsymbol{\Sigma}}_{h}^{k}$ and all $T \in \mathcal{T}_{h}$,

$$
D_{h}^{k} \boldsymbol{\tau}_{h \mid T}=D_{T}^{k} R_{\Sigma, T}^{k} \boldsymbol{\tau}_{h} .
$$

Using the definition (15) of the global interpolator $I_{\Sigma, h}^{k}$ together with the commuting property (18) for the local divergence operator, the following global commuting property follows $\left(\nabla_{h}\right.$. denotes here the broken divergence operator on $\left.\mathcal{T}_{h}\right)$ :

$$
D_{h}^{k}\left(I_{\boldsymbol{\Sigma}, h}^{k} \boldsymbol{t}\right)=\pi_{h}^{k}\left(\boldsymbol{\nabla}_{h} \cdot \boldsymbol{t}\right), \quad \forall \boldsymbol{t} \in \boldsymbol{\Sigma}^{+} .
$$

We next introduce the flux reconstruction operator $\mathfrak{C}_{T}^{k}: \Sigma_{T}^{k} \rightarrow \nabla \mathbb{P}_{d}^{k+1}(T)$ such that, for all $\boldsymbol{\tau}=\left(\boldsymbol{\tau}_{T},\left(\tau_{T F}\right)_{F \in \mathcal{F}_{T}}\right) \in \boldsymbol{\Sigma}_{T}^{k}$ and, for all $w \in \mathbb{P}_{d}^{k+1}(T)$,

$$
\begin{aligned}
\left(\mathfrak{C}_{T}^{k} \boldsymbol{\tau}, \boldsymbol{\nabla} w\right)_{T} & =-\left(D_{T}^{k} \boldsymbol{\tau}, w\right)_{T}+\sum_{F \in \mathcal{F}_{T}}\left(\tau_{T F}, w\right)_{F} \\
& =\left(\boldsymbol{\tau}_{T}, \boldsymbol{\nabla} \pi_{T}^{k} w\right)_{T}+\sum_{F \in \mathcal{F}_{T}}\left(\tau_{T F}, \pi_{F}^{k} w-\pi_{T}^{k} w\right)_{F},
\end{aligned}
$$

where we have used $D_{T}^{k} \boldsymbol{\tau} \in \mathbb{P}_{d}^{k}(T)$ together with (17) to pass to the second line. Computing $y \in$ $\mathbb{P}_{d}^{k+1}(T)$ such that $\mathfrak{C}_{T}^{k} \boldsymbol{\tau}=\boldsymbol{\nabla} y$ and (21) holds requires to solve a well-posed Neumann problem for which the usual compatibility condition on the right-hand side is verified. The following polynomial consistency property for $\mathfrak{C}_{T}^{k}$ is an immediate consequence of (21a) recalling (17) and (14):

$$
\mathfrak{C}_{T}^{k} I_{\Sigma, T}^{k} \nabla w=\nabla w, \quad \forall w \in \mathbb{P}_{d}^{k+1}(T) .
$$

Recalling [19, Lemma 3] and using the fact that $\left\|D_{T}^{k} \boldsymbol{\tau}\right\|_{T} \leqslant C h_{T}^{-1}\|\boldsymbol{\tau}\|_{T}$, we have continuity and partial stability in the following sense: For all $\boldsymbol{\tau}=\left(\boldsymbol{\tau}_{T},\left(\tau_{T F}\right)_{F \in \mathcal{F}_{T}}\right) \in \boldsymbol{\Sigma}_{T}^{k}$,

$$
\left\|\boldsymbol{\tau}_{T}\right\|_{T} \leqslant\left\|\mathfrak{C}_{T}^{k} \boldsymbol{\tau}\right\|_{T} \leqslant\|\boldsymbol{\tau}\|_{T} .
$$




\subsubsection{Mixed formulation}

We let $H$ denote a global bilinear form on $\check{\Sigma}_{h}^{k} \times \check{\boldsymbol{\Sigma}}_{h}^{k}$ assembled element-wise from local contributions, i.e., such that, for all $\boldsymbol{\sigma}_{h}, \boldsymbol{\tau}_{h} \in \check{\Sigma}_{h}^{k}$,

$$
H\left(\boldsymbol{\sigma}_{h}, \boldsymbol{\tau}_{h}\right):=\sum_{T \in \mathcal{T}_{h}} H_{T}\left(R_{\Sigma, T}^{k} \boldsymbol{\sigma}_{h}, R_{\Sigma, T}^{k} \boldsymbol{\tau}_{h}\right),
$$

where, for all $T \in \mathcal{T}_{h}$, the bilinear form $H_{T}$ on $\boldsymbol{\Sigma}_{T}^{k} \times \boldsymbol{\Sigma}_{T}^{k}$ is such that, for all $\boldsymbol{\sigma}, \boldsymbol{\tau} \in \boldsymbol{\Sigma}_{T}^{k}$,

$$
H_{T}(\boldsymbol{\sigma}, \boldsymbol{\tau}):=\left(\mathfrak{C}_{T}^{k} \boldsymbol{\sigma}, \mathfrak{C}_{T}^{k} \boldsymbol{\tau}\right)_{T}+J_{T}(\boldsymbol{\sigma}, \boldsymbol{\tau}),
$$

with local stabilization bilinear form $J_{T}$ matching the following assumptions:

(H1) Nonnegativity and polynomial consistency. $J_{T}$ is symmetric, positive semi-definite, and it satisfies the following polynomial consistency condition:

$$
\forall w \in \mathbb{P}_{d}^{k+1}(T), \quad J_{T}\left(I_{\boldsymbol{\Sigma}, T}^{k} \boldsymbol{\nabla} w, \boldsymbol{\tau}\right)=0 \quad \forall \boldsymbol{\tau} \in \boldsymbol{\Sigma}_{T}^{k} .
$$

(H2) Stability and continuity. There exists a real number $\eta>0$ independent of $T$ and of $h$ such that $H_{T}$ is coercive on $\operatorname{ker}\left(D_{T}^{k}\right)$ and continuous on $\boldsymbol{\Sigma}_{T}^{k}$ :

$$
\begin{array}{cc}
\eta\|\boldsymbol{\tau}\|_{T}^{2} \leqslant H_{T}(\boldsymbol{\tau}, \boldsymbol{\tau}) & \forall \boldsymbol{\tau} \in \operatorname{ker}\left(D_{T}^{k}\right), \\
H_{T}(\boldsymbol{\tau}, \boldsymbol{\tau}) \leqslant \eta^{-1}\|\boldsymbol{\tau}\|_{T}^{2} & \forall \boldsymbol{\tau} \in \boldsymbol{\Sigma}_{T}^{k} .
\end{array}
$$

Remark 2 (Condition (26b)). In view of (24) and of the second inequality in (23), and since $J_{T}$ is symmetric and positive semi-definite owing to (H1), condition (26b) holds if and only if there is a real number $C>0$ independent of $h$ such that, for all $T \in \mathcal{T}_{h}$,

$$
J_{T}(\boldsymbol{\tau}, \boldsymbol{\tau}) \leqslant C\|\boldsymbol{\tau}\|_{T}^{2} \quad \forall \boldsymbol{\tau} \in \mathbf{\Sigma}_{T}^{k} .
$$

An example for a stabilization bilinear form satisfying assumptions (H1)-(H2) is

$$
J_{T}(\boldsymbol{\sigma}, \boldsymbol{\tau}):=\sum_{F \in \mathcal{F}_{T}} h_{F}\left(\mathfrak{C}_{T}^{k} \boldsymbol{\sigma} \cdot \boldsymbol{n}_{T F}-\sigma_{T F}, \mathfrak{C}_{T}^{k} \boldsymbol{\tau} \cdot \boldsymbol{n}_{T F}-\tau_{T F}\right)_{F}
$$

For further use, we also define the global stabilization bilinear form $J$ on $\check{\Sigma}_{h}^{k} \times \breve{\Sigma}_{h}^{k}$ such that

$$
J\left(\boldsymbol{\sigma}_{h}, \boldsymbol{\tau}_{h}\right):=\sum_{T \in \mathcal{T}_{h}} J_{T}\left(R_{\boldsymbol{\Sigma}, T}^{k} \boldsymbol{\sigma}_{h}, R_{\boldsymbol{\Sigma}, T}^{k} \boldsymbol{\tau}_{h}\right)
$$

Letting $f_{h}:=\pi_{h}^{k} f$, the mixed discrete problem reads: Find $\left(\boldsymbol{\sigma}_{h}, u_{h}\right) \in \boldsymbol{\Sigma}_{h}^{k} \times U_{h}^{k}$ such that

$$
\begin{aligned}
H\left(\boldsymbol{\sigma}_{h}, \boldsymbol{\tau}_{h}\right)+\left(u_{h}, D_{h}^{k} \boldsymbol{\tau}_{h}\right) & =0 & & \forall \boldsymbol{\tau}_{h} \in \boldsymbol{\Sigma}_{h}^{k}, \\
-\left(D_{h}^{k} \boldsymbol{\sigma}_{h}, v_{h}\right) & =\left(f_{h}, v_{h}\right) & & \forall v_{h} \in U_{h}^{k} .
\end{aligned}
$$

The well-posedness of problem (30) is a classical consequence of the coercivity (26a) of $H_{T}$ in the kernel of $D_{T}^{k}$ together with the commuting property (20). 


\subsection{Mixed hybrid formulation}

In this section we hybridize (30) in the spirit of [2] by using the unpatched space $\breve{\mathbf{\Sigma}}_{h}^{k}$ defined by (10) in place of the subspace $\boldsymbol{\Sigma}_{h}^{k}$ defined by (11), and we enforce the single-valuedness of flux DOFs located at interfaces via Lagrange multipliers. Let

$$
\Lambda_{h}^{k}:=\underset{F \in \mathcal{F}_{h}}{X} \Lambda_{F}^{k} \quad \text { with } \quad \Lambda_{F}^{k}:= \begin{cases}\mathbb{P}_{d-1}^{k}(F) & \text { if } F \in \mathcal{F}_{h}^{\mathrm{i}}, \\ \{0\} & \text { if } F \in \mathcal{F}_{h}^{\mathrm{b}},\end{cases}
$$

and define the following local and global hybrid DOF spaces $\left(U_{T}^{k}\right.$ and $U_{h}^{k}$ are given by (16)):

$$
W_{T}^{k}:=U_{T}^{k} \times\left\{\underset{F \in \mathcal{F}_{T}}{X} \Lambda_{F}^{k}\right\} \quad \forall T \in \mathcal{T}_{h} \quad \text { and } \quad W_{h}^{k}:=U_{h}^{k} \times \Lambda_{h}^{k} .
$$

We introduce the bilinear form $B$ on $\breve{\boldsymbol{\Sigma}}_{h}^{k} \times W_{h}^{k}$ such that, for all $\left(\boldsymbol{\tau}_{h}, z_{h}\right) \in \breve{\boldsymbol{\Sigma}}_{h}^{k} \times W_{h}^{k}$ with $z_{h}=\left(v_{h}, \mu_{h}\right)$, recalling (17) and (19) to infer the second equality,

$$
\begin{aligned}
B\left(\boldsymbol{\tau}_{h}, z_{h}\right) & :=\left(v_{h}, D_{h}^{k} \boldsymbol{\tau}_{h}\right)-\sum_{T \in \mathcal{T}_{h}} \sum_{F \in \mathcal{F}_{T}}\left(\mu_{F}, \tau_{T F}\right)_{F} \\
& =\sum_{T \in \mathcal{T}_{h}}\left\{-\left(\boldsymbol{\nabla} v_{T}, \boldsymbol{\tau}_{T}\right)_{T}+\sum_{F \in \mathcal{F}_{T}}\left(v_{T}-\mu_{F}, \tau_{T F}\right)_{F}\right\} .
\end{aligned}
$$

Problem (30) reformulates as follows: Find $\overline{\boldsymbol{\sigma}}_{h} \in \check{\boldsymbol{\Sigma}}_{h}^{k}$ and $\bar{w}_{h}:=\left(\bar{u}_{h}, \lambda_{h}\right) \in W_{h}^{k}$ such that,

$$
\begin{aligned}
H\left(\overline{\boldsymbol{\sigma}}_{h}, \boldsymbol{\tau}_{h}\right)+B\left(\boldsymbol{\tau}_{h}, \bar{w}_{h}\right) & =0 & & \forall \boldsymbol{\tau}_{h} \in \check{\Sigma}_{h}^{k}, \\
-B\left(\overline{\boldsymbol{\sigma}}_{h}, z_{h}\right) & =\left(f_{h}, v_{h}\right) & & \forall z_{h}=\left(v_{h}, \mu_{h}\right) \in W_{h}^{k} .
\end{aligned}
$$

The following result justifies the choice of the space (31) by showing that problem (34) is well-posed, and establishes a link between the solutions of (30) and (34).

Lemma 3 (Relation between (30) and (34)). The following inf-sup condition holds with $C>0$ independent of $h$

$$
C\left\|z_{h}\right\|_{0, h} \leqslant \sup _{\boldsymbol{\tau}_{h} \in \breve{\boldsymbol{\Sigma}}_{h}^{k},\left\|\boldsymbol{\tau}_{h}\right\|=1} B\left(\boldsymbol{\tau}_{h}, z_{h}\right)
$$

where, for all $z_{h}=\left(v_{h}, \mu_{h}\right) \in W_{h}^{k},\left\|z_{h}\right\|_{0, h}^{2}:=\left\|v_{h}\right\|^{2}+\sum_{T \in \mathcal{T}_{h}} \sum_{F \in \mathcal{F}_{T}} h_{F}^{-1}\left\|\mu_{F}-v_{T}\right\|_{F}^{2} . \quad$ Additionally, problem (34) has a unique solution $\left(\overline{\boldsymbol{\sigma}}_{h},\left(\bar{u}_{h}, \lambda_{h}\right)\right) \in \check{\mathbf{\Sigma}}_{h}^{k} \times W_{h}^{k}$. Finally, denoting by $\left(\boldsymbol{\sigma}_{h}, u_{h}\right) \in \boldsymbol{\Sigma}_{h}^{k} \times U_{h}^{k}$ the unique solution to problem $(30)$, it holds $\left(\overline{\boldsymbol{\sigma}}_{h}, \bar{u}_{h}\right)=\left(\boldsymbol{\sigma}_{h}, u_{h}\right)$. In view of this result, we drop the bar in what follows.

Proof. Let $z_{h}=\left(v_{h}, \mu_{h}\right) \in W_{h}^{k}$. Following [10], there is $\boldsymbol{t}_{v} \in \boldsymbol{\Sigma}^{+} \cap \boldsymbol{H}(\operatorname{div} ; \Omega)$ such that $\boldsymbol{\nabla} \cdot \boldsymbol{t}_{v}=v_{h}$. Letting $\boldsymbol{\tau}_{h, 1}:=I_{\boldsymbol{\Sigma}, h}^{k} \boldsymbol{t}_{v} \in \boldsymbol{\Sigma}_{h}^{k}$ (recall Remark 1), using the continuity of $I_{\boldsymbol{\Sigma}, h}^{k}$ and of $v_{h} \mapsto \boldsymbol{t}_{v}$, it holds $\left\|\boldsymbol{\tau}_{h, 1}\right\| \lesssim\left\|\boldsymbol{t}_{v}\right\|_{\boldsymbol{\Sigma}^{+}} \lesssim\left\|v_{h}\right\|$ and, owing to the commuting property (20), $\left\|v_{h}\right\|^{2}=$ $\left(v_{h}, D_{h}^{k} \boldsymbol{\tau}_{h, 1}\right)=B\left(\boldsymbol{\tau}_{h, 1}, z_{h}\right)$ since $\sum_{T \in \mathcal{T}_{h}} \sum_{F \in \mathcal{F}_{T}}\left(\mu_{F}, \boldsymbol{\tau}_{T F, 1}\right)_{F}=0$ again as a consequence of having $\boldsymbol{\tau}_{h, 1} \in \boldsymbol{\Sigma}_{h}^{k}$. Moreover, letting $\boldsymbol{\tau}_{h, 2} \in \check{\boldsymbol{\Sigma}}_{h}^{k}$ be such that $\boldsymbol{\tau}_{T, 2} \equiv \mathbf{0}$ and $\tau_{T F, 2}=h_{F}^{-1}\left(v_{T}-\mu_{F}\right)$ for all $T \in \mathcal{T}_{h}$ and $F \in \mathcal{F}_{T}$, one has, recalling (33b), $B\left(\boldsymbol{\tau}_{h, 2}, z_{h}\right)=\sum_{T \in \mathcal{T}_{h}} \sum_{F \in \mathcal{F}_{T}} h_{F}^{-1}\left\|\mu_{F}-v_{T}\right\|_{F}^{2}$. 
Additionally, it clearly holds that $\left\|\boldsymbol{\tau}_{h, 2}\right\| \lesssim\left\|z_{h}\right\|_{0, h}$. As a result, using the linearity of $B$ in its first argument, one has, denoting by $\$$ the supremum in the right-hand side of (35),

$$
\left\|z_{h}\right\|_{0, h}^{2}=B\left(\boldsymbol{\tau}_{h, 1}+\boldsymbol{\tau}_{h, 2}, z_{h}\right) \leqslant \$\left\|\boldsymbol{\tau}_{h, 1}+\boldsymbol{\tau}_{h, 2}\right\| \lesssim \$\left\|z_{h}\right\|_{0, h}
$$

and (35) follows. The well-posedness of problem (34) is a consequence of (35) together with the coercivity of the bilinear form $H$ in the kernel of $D_{h}^{k}$, cf. (26a), see [10]. To prove the last part of the statement, we observe that the $\left(\boldsymbol{\sigma}_{h},\left(u_{h}, 0\right)\right) \in \check{\boldsymbol{\Sigma}}_{h}^{k} \times W_{h}^{k}$ is clearly a solution to problem (34) since (34a) with $\lambda_{h}=0$ and (34b) with $z_{h}=\left(v_{h}, 0\right)$ follow from (30a) and (30b), respectively, while $(34 \mathrm{~b})$ with $z_{h}=\left(0, \mu_{h}\right)$, which enforces, $\sum_{T \in \mathcal{T}_{F}} \bar{\sigma}_{T F}=0$ for all $F \in \mathcal{F}_{h}^{\mathrm{i}}$, holds true if $\overline{\boldsymbol{\sigma}}_{h}=\boldsymbol{\sigma}_{h} \in \boldsymbol{\Sigma}_{h}^{k}$, cf. (11). On the other hand, since problem (34) is well-posed, it must hold that $\left(\overline{\boldsymbol{\sigma}}_{h}, \bar{u}_{h}\right)=\left(\boldsymbol{\sigma}_{h}, u_{h}\right)$, which concludes the proof.

\subsection{Primal hybrid formulation}

In this section, we reformulate the mixed hybrid problem (34) as a coercive primal hybrid problem after locally eliminating the flux DOFs, and we establish a link with HHO methods.

We need, in what follows, a $H_{0}^{1}$-like discrete norm as well as an interpolator on the space of hybrid DOFs, cf (32). For all $T \in \mathcal{T}_{h}$, denote by $R_{W, T}^{k}: W_{h}^{k} \rightarrow W_{T}^{k}$ the restriction operator that maps global to local DOFs. We equip $W_{h}^{k}$ with the norm such that, for all $z_{h} \in W_{h}^{k}$,

$$
\left\|z_{h}\right\|_{1, h}^{2}:=\sum_{T \in \mathcal{T}_{h}}\left\|R_{W, T}^{k} z_{h}\right\|_{1, T}^{2}
$$

with local norm such that, for all $z=\left(v_{T},\left(\mu_{F}\right)_{F \in \mathcal{F}_{T}}\right) \in W_{T}^{k}$,

$$
\|z\|_{1, T}^{2}:=\left\|\nabla v_{T}\right\|_{T}^{2}+\sum_{F \in \mathcal{F}_{T}} h_{F}^{-1}\left\|\mu_{F}-v_{T}\right\|_{F}^{2} \quad \forall T \in \mathcal{T}_{h} .
$$

One can easily prove that the map defined by (36) is a norm on $W_{h}^{k}$ using the fact that Lagrange multipliers are zero at boundary faces, cf. (31). Let

$$
W(T):=\left\{v \in H^{1}(T) \mid v_{\mid \partial T \cap \partial \Omega} \equiv 0\right\} .
$$

We introduce the local interpolator $I_{W, T}^{k}: W(T) \rightarrow W_{T}^{k}$ such that, for all $v \in W(T)$,

$$
I_{W, T}^{k} v=\left(v_{T},\left(\mu_{F}\right)_{F \in \mathcal{F}_{T}}\right) \quad \text { with } \quad v_{T}=\pi_{T}^{k} v \quad \text { and } \quad \mu_{F}=\pi_{F}^{k} v \quad \forall F \in \mathcal{F}_{T} .
$$

The corresponding global interpolator is $I_{W, h}^{k}: W \rightarrow W_{h}^{k}$ (recall that $W:=H_{0}^{1}(\Omega)$ ) such that, for all $v \in W$,

$$
I_{W, h}^{k} v=\left(\left(v_{T}\right)_{T \in \mathcal{T}_{h}},\left(\mu_{F}\right)_{F \in \mathcal{F}_{h}}\right) \quad \text { with } \quad v_{T}=\pi_{T}^{k} v \quad \forall T \in \mathcal{T}_{h} \quad \text { and } \quad \mu_{F}=\pi_{F}^{k} v \quad \forall F \in \mathcal{F}_{h} .
$$




\subsubsection{Potential lifting operator}

A first step consists in defining local and global operators which allow, given a set of potential DOFs, to identify the corresponding flux DOFs. We need from this point on a stronger assumption than (26a), namely:

$$
\eta\|\boldsymbol{\tau}\|_{T}^{2} \leqslant H_{T}(\boldsymbol{\tau}, \boldsymbol{\tau}) \quad \forall \boldsymbol{\tau} \in \boldsymbol{\Sigma}_{T}^{k},
$$

so that $H_{T}($ resp. $H)$ is actually an inner-product on $\boldsymbol{\Sigma}_{T}^{k}$ (resp. $\breve{\boldsymbol{\Sigma}}_{h}^{k}$ ), defining a norm $\|\cdot\|_{H, T}$ (resp. $\|\cdot\|_{H}$ ) equivalent to $\|\cdot\|_{T}$ (resp. $\|\cdot\| \|$ ). Let us check that the stabilization bilinear form $J_{T}$ defined by (28) satisfies $\left(\mathbf{H 2}^{+}\right)$. Recalling the first inequality in (23) to infer $\left\|\boldsymbol{\tau}_{T}\right\|_{T} \leqslant\left\|\mathfrak{C}_{T}^{k} \boldsymbol{\tau}\right\|_{T}$, and introducing the quantity $\mathfrak{C}_{T}^{k} \boldsymbol{\tau} \cdot \boldsymbol{n}_{T F}$ in the second term in the right-hand side of (12), one has, for all $\boldsymbol{\tau} \in \boldsymbol{\Sigma}_{T}^{k}$,

$$
\begin{aligned}
\|\boldsymbol{\tau}\|_{T}^{2} & \lesssim\left\|\mathfrak{C}_{T}^{k} \boldsymbol{\tau}\right\|_{T}^{2}+\sum_{F \in \mathcal{F}_{T}} h_{F}\left\|\mathfrak{C}_{T}^{k} \boldsymbol{\tau} \cdot \boldsymbol{n}_{T F}-\tau_{T F}\right\|_{F}^{2}+\sum_{F \in \mathcal{F}_{T}} h_{F}\left\|\mathfrak{C}_{T}^{k} \boldsymbol{\tau} \cdot \boldsymbol{n}_{T F}\right\|_{F}^{2} \\
& \lesssim\left\|\mathfrak{C}_{T}^{k} \boldsymbol{\tau}\right\|_{T}^{2}+J_{T}(\boldsymbol{\tau}, \boldsymbol{\tau})=H_{T}(\boldsymbol{\tau}, \boldsymbol{\tau}),
\end{aligned}
$$

where we have used the definition (28) of $J_{T}$ together with the discrete trace inequality (2) and the bound (1) on $\mathfrak{N}_{T}$ to pass from the first to the second line, plus the definition (24) of the bilinear form $H_{T}$ to conclude.

For all $T \in \mathcal{T}_{h}$, a local potential lifting operator $\boldsymbol{\varsigma}_{T}^{k}: W_{T}^{k} \rightarrow \boldsymbol{\Sigma}_{T}^{k}$ can be naturally defined such that, for all $z=\left(v_{T},\left(\mu_{F}\right)_{F \in \mathcal{F}_{T}}\right) \in W_{T}^{k}$, it holds, for all $\boldsymbol{\tau} \in \boldsymbol{\Sigma}_{T}^{k}$,

$$
\begin{aligned}
H_{T}\left(\boldsymbol{\varsigma}_{T}^{k} z, \boldsymbol{\tau}\right) & =-\left(v_{T}, D_{T}^{k} \boldsymbol{\tau}\right)_{T}+\sum_{F \in \mathcal{F}_{T}}\left(\mu_{F}, \tau_{T F}\right)_{F} \\
& =\left(\boldsymbol{\nabla} v_{T}, \boldsymbol{\tau}_{T}\right)_{T}+\sum_{F \in \mathcal{F}_{T}}\left(\mu_{F}-v_{T}, \tau_{T F}\right)_{F},
\end{aligned}
$$

insofar as this yields a well-posed problem for $\boldsymbol{\varsigma}_{T}^{k} z$ in view of $\left(\mathbf{H 2}^{+}\right)$(we have used the definition (17) of $D_{T}^{k}$ to pass to the second line). We also define the global lifting operator $\varsigma_{h}^{k}: W_{h}^{k} \rightarrow \check{\Sigma}_{h}^{k}$ such that, for all $z_{h} \in W_{h}^{k}$,

$$
R_{\Sigma, T}^{k} \varsigma_{h}^{k} z_{h}=\varsigma_{T}^{k} R_{W, T}^{k} z_{h} \quad \forall T \in \mathcal{T}_{h} .
$$

An important remark is that, as a consequence of (41a), $\boldsymbol{\varsigma}_{h}^{k} z_{h}$ satisfies

$$
\forall z_{h} \in W_{h}^{k}, \quad H\left(\boldsymbol{\varsigma}_{h}^{k} z_{h}, \boldsymbol{\tau}_{h}\right)=-B\left(\boldsymbol{\tau}_{h}, z_{h}\right) \quad \forall \boldsymbol{\tau}_{h} \in \check{\boldsymbol{\Sigma}}_{h}^{k},
$$

with bilinear form $B$ defined by (33a).

Lemma 4 (Stability and continuity for $\boldsymbol{\varsigma}_{T}^{k}$ ). For all $T \in \mathcal{T}_{h}$ and all $z \in W_{T}^{k}$, it holds, denoting by $\|\cdot\|_{H, T}$ the norm defined by $H_{T}$ on $\boldsymbol{\Sigma}_{T}^{k}$,

$$
\eta^{1 / 2}\|z\|_{1, T} \leqslant\left\|\boldsymbol{\varsigma}_{T}^{k} z\right\|_{H, T} \leqslant \eta^{-1 / 2}\|z\|_{1, T} .
$$

Thus, for all $z_{h} \in W_{h}^{k}$, we have, with $\|\cdot\|_{H}$ denoting the norm defined by $H$ on $\check{\mathbf{\Sigma}}_{h}^{k}$,

$$
\eta^{1 / 2}\left\|z_{h}\right\|_{1, h} \leqslant\left\|\boldsymbol{\varsigma}_{h}^{k} z_{h}\right\|_{H} \leqslant \eta^{-1 / 2}\left\|z_{h}\right\|_{1, h} .
$$


Proof. Let $z=\left(v_{T},\left(\mu_{F}\right)_{F \in \mathcal{F}_{T}}\right) \in W_{T}^{k}$. Letting $\boldsymbol{\tau}_{z}=\left(\boldsymbol{\nabla}\left(v_{T}-\pi_{T}^{0} v_{T}\right),\left(h_{F}^{-1}\left(\mu_{F}-v_{T}\right)\right)_{F \in \mathcal{F}_{T}}\right) \in \boldsymbol{\Sigma}_{T}^{k}$ so that $\left\|\boldsymbol{\tau}_{z}\right\|_{T}=\|z\|_{1, T}$, one has, using (41b) with $\boldsymbol{\tau}=\boldsymbol{\tau}_{z}$ followed by (26b), $H_{T}\left(\boldsymbol{\varsigma}_{T}^{k} z, \boldsymbol{\tau}_{z}\right)=$ $\|z\|_{1, T}^{2}=\left\|\boldsymbol{\tau}_{z}\right\|_{T}\|z\|_{1, T} \geqslant \eta^{1 / 2}\left\|\boldsymbol{\tau}_{z}\right\|_{H, T}\|z\|_{1, T}$. Hence, to prove the first inequality in (43), observe that $\eta^{1 / 2}\|z\|_{1, T} \leqslant \sup _{\boldsymbol{\tau} \in \boldsymbol{\Sigma}_{T}^{k} \backslash\{\mathbf{0}\}} \frac{H_{T}\left(\boldsymbol{\varsigma}_{T}^{k} z, \boldsymbol{\tau}\right)}{\|\boldsymbol{\tau}\|_{H, T}}=\left\|\boldsymbol{\varsigma}_{T}^{k} z\right\|_{H, T}$, since $H_{T}$ defines an inner-product. On the other hand, it holds for all $\boldsymbol{\tau} \in \boldsymbol{\Sigma}_{T}^{k}$, bounding the right-hand side of (41b) with the Cauchy-Schwarz inequality, and recalling the definitions (37) of the $\|\cdot\|_{1, T}$-norm and (12) of the $\|\cdot\|_{T}$-norm, $H_{T}\left(\boldsymbol{\varsigma}_{T}^{k} z, \boldsymbol{\tau}\right) \leqslant\|z\|_{1, T}\|\boldsymbol{\tau}\|_{T} \leqslant \eta^{-1 / 2}\|z\|_{1, T}\|\boldsymbol{\tau}\|_{H, T}$, where we have used $\left(\mathbf{H 2}^{+}\right)$ to conclude. The second inequality in (43) then follows from the previous bound observing that $\left\|\boldsymbol{\varsigma}_{T}^{k} z\right\|_{H, T}=\sup _{\boldsymbol{\tau} \in \boldsymbol{\Sigma}_{T}^{k}}\left\{H_{T}\left(\boldsymbol{\varsigma}_{T}^{k} z, \boldsymbol{\tau}\right) /\|\boldsymbol{\tau}\|_{H, T}\right\}$. Finally, (44) can be proved squaring (43) and summing over $T \in \mathcal{T}_{h}^{T}$.

\subsubsection{Discrete gradient and potential reconstruction operators}

Let us next define the consistent gradient reconstruction operator

$$
\boldsymbol{G}_{T}^{k}:=\mathfrak{C}_{T}^{k} \circ \boldsymbol{\varsigma}_{T}^{k}
$$

with $\mathfrak{C}_{T}^{k}$ and $\boldsymbol{\varsigma}_{T}^{k}$ defined by (21) and (41), respectively. The consistent gradient satisfies the following remarkable property: For all $z=\left(v_{T},\left(\mu_{F}\right)_{F \in \mathcal{F}_{T}}\right) \in W_{T}^{k}$,

$$
\left(\boldsymbol{G}_{T}^{k} z, \boldsymbol{\nabla} w\right)_{T}=\left(\boldsymbol{\nabla} v_{T}, \boldsymbol{\nabla} w\right)_{T}+\sum_{F \in \mathcal{F}_{T}}\left(\mu_{F}-v_{T}, \boldsymbol{\nabla} w \cdot \boldsymbol{n}_{T F}\right)_{F} \quad \forall w \in \mathbb{P}_{d}^{k+1}(T) .
$$

To prove (46), let $w \in \mathbb{P}_{d}^{k+1}(T)$ be fixed, make $\boldsymbol{\tau}:=I_{\boldsymbol{\Sigma}, T}^{k} \boldsymbol{\nabla} w$ in (41b), and use the fact that $\mathfrak{C}_{T}^{k} \boldsymbol{\tau}=\boldsymbol{\nabla} w$ owing to $(22)$ and that $\mathfrak{C}_{T}^{k} \boldsymbol{\varsigma}_{T}^{k} z=\boldsymbol{G}_{T}^{k} z$ and $J_{T}\left(\boldsymbol{\varsigma}_{T}^{k} z, \boldsymbol{\tau}\right)=J_{T}\left(\boldsymbol{\varsigma}_{T}^{k} z, I_{\boldsymbol{\Sigma}, T}^{k} \boldsymbol{\nabla} w\right)=0$ owing to (45) and (25), respectively, to infer from the definition (24) of $H_{T}$ that

$$
H_{T}\left(\boldsymbol{\varsigma}_{T}^{k} z, \boldsymbol{\tau}\right)=\left(\mathfrak{C}_{T}^{k} \boldsymbol{\varsigma}_{T}^{k} z, \mathfrak{C}_{T}^{k} \boldsymbol{\tau}\right)+J_{T}\left(\boldsymbol{\varsigma}_{T}^{k} z, \boldsymbol{\tau}\right)=\left(\boldsymbol{G}_{T}^{k} z, \nabla w\right)_{T}
$$

We remark at this point an important result: equation (46) shows that the discrete gradient operator defined by (45) is in fact analogous to the one defined in [21, eq. (11)] in the framework of HHO methods provided the Lagrange multipliers are interpreted as trace unknowns. In what follows we recall some important consequences without proof.

(i) For any function $\varphi \in W(T)$, the following orthogonality property holds:

$$
\left(\boldsymbol{G}_{T}^{k} I_{W, T}^{k} \varphi-\nabla \varphi, \nabla w\right)_{T}=0 \quad \forall w \in \mathbb{P}_{d}^{k+1}(T) .
$$

(ii) For all $z \in W_{T}^{k}$, it holds, denoting by $\left(\boldsymbol{\varsigma}_{T}^{k} z\right)_{T} \in \mathbb{T}_{T}^{k}$ the cell DOFs for $\boldsymbol{\varsigma}_{T}^{k} z \in \boldsymbol{\Sigma}_{T}^{k}$,

$$
\left\|\left(\boldsymbol{\varsigma}_{T}^{k} z\right)_{T}\right\|_{T} \leqslant\left\|\boldsymbol{G}_{T}^{k} z\right\|_{T} \leqslant\left\|\boldsymbol{\varsigma}_{T}^{k} z\right\|_{H, T} \leqslant \eta^{-1 / 2}\|z\|_{1, T} \quad \forall z \in W_{T}^{k} .
$$

(iii) Defining, for all $T \in \mathcal{T}_{h}$, the local potential reconstruction operator $r_{T}^{k}: W_{T}^{k} \rightarrow \mathbb{P}_{d}^{k+1}(T)$ such that, for all $z=\left(v_{T},\left(\mu_{F}\right)_{F \in \mathcal{F}_{T}}\right) \in W_{T}^{k}$,

$$
\nabla r_{T}^{k} z=G_{T}^{k} z, \quad \int_{T} r_{T}^{k} z=\int_{T} v_{T}
$$


there exists a real number $C>0$, independent of $h_{T}$ such that, for all $v \in W(T) \cap$ $H^{k+2}(T)$,

$$
\begin{aligned}
h_{T}\left\|\boldsymbol{\nabla}\left(v-r_{T}^{k} I_{W, T}^{k} v\right)\right\|_{T}+h_{T}^{3 / 2}\left\|\boldsymbol{\nabla}\left(v-r_{T}^{k} I_{W, T}^{k} v\right)\right\|_{\partial T} \\
\quad+\left\|v-r_{T}^{k} I_{W, T}^{k} v\right\|_{T}+h_{T}^{1 / 2}\left\|v-r_{T}^{k} I_{W, T}^{k} v\right\|_{\partial T} \leqslant C h_{T}^{k+2}\|v\|_{H^{k+2}(T)} .
\end{aligned}
$$

We close this section by defining global gradient and potential reconstructions as follows: For all $z_{h} \in W_{h}^{k}$, we let

$$
\boldsymbol{G}_{h}^{k} z_{h \mid T}:=\boldsymbol{G}_{T}^{k} R_{W, T}^{k} z_{h} \quad \text { and } \quad r_{h}^{k} z_{h \mid T}=r_{T}^{k} R_{W, T}^{k} z_{h} \quad \forall T \in \mathcal{T}_{h} .
$$

\subsubsection{Primal hybrid formulation}

Denoting by $\left(\boldsymbol{\sigma}_{h}, w_{h}\right) \in \breve{\boldsymbol{\Sigma}}_{h}^{k} \times W_{h}^{k}$ the solution to problem (34) (we have removed the bar from $\boldsymbol{\sigma}_{h}$ as a result of Lemma 3), it is readily inferred from (42) and (34a) that

$$
\boldsymbol{\sigma}_{h}=\boldsymbol{\varsigma}_{h}^{k} w_{h}
$$

Then, using (52), equation (34b) can be rewritten for all $z_{h}=\left(v_{h}, \mu_{h}\right) \in W_{h}^{k}$ as

$$
-B\left(\boldsymbol{\varsigma}_{h}^{k} w_{h}, z_{h}\right)=\left(f_{h}, v_{h}\right) .
$$

Define the bilinear form $A$ on $W_{h}^{k} \times W_{h}^{k}$ such that, for all $w_{h}, z_{h} \in W_{h}^{k}$,

$$
A\left(w_{h}, z_{h}\right):=H\left(\boldsymbol{\varsigma}_{h}^{k} w_{h}, \boldsymbol{\varsigma}_{h}^{k} z_{h}\right)=\left(\boldsymbol{G}_{h}^{k} w_{h}, \boldsymbol{G}_{h}^{k} z_{h}\right)+j\left(w_{h}, z_{h}\right),
$$

where we have introduced the bilinear form $j$ on $W_{h}^{k} \times W_{h}^{k}$ such that ( $J$ is defined by (29)),

$$
j\left(w_{h}, z_{h}\right):=J\left(\boldsymbol{\varsigma}_{h}^{k} w_{h}, \boldsymbol{\varsigma}_{h}^{k} z_{h}\right) .
$$

The equality in (53) is a straightforward consequence of (24) together with (41b). Then, recalling (42) and using the symmetry of the bilinear form $H$, it is inferred, for all $z_{h} \in W_{h}^{k}$,

$$
-B\left(\boldsymbol{\varsigma}_{h}^{k} w_{h}, z_{h}\right)=H\left(\boldsymbol{\varsigma}_{h}^{k} w_{h}, \boldsymbol{\varsigma}_{h}^{k} z_{h}\right)=A\left(w_{h}, z_{h}\right),
$$

and we conclude that problem (34) can be reformulated as follows: Find $w_{h}=\left(u_{h}, \lambda_{h}\right) \in W_{h}^{k}$ such that,

$$
A\left(w_{h}, z_{h}\right)=\left(f, v_{h}\right) \quad \forall z_{h}=\left(v_{h}, \mu_{h}\right) \in W_{h}^{k},
$$

and (52) holds. It follows from (44) that, for all $z_{h} \in W_{h}^{k}$, observing that $A\left(z_{h}, z_{h}\right)=\left\|\boldsymbol{\varsigma}_{h}^{k} z_{h}\right\|_{H, T}^{2}$ as a consequence of (53),

$$
\eta\left\|z_{h}\right\|_{1, h}^{2} \leqslant A\left(z_{h}, z_{h}\right):=\left\|z_{h}\right\|_{A}^{2} \leqslant \eta^{-1}\left\|z_{h}\right\|_{1, h}^{2} .
$$

As a result, the bilinear form $A$ is coercive, and the well-posedness of the new problem (55) follows directly from the Lax-Milgram lemma.

From a practical viewpoint, the symmetric positive definite linear system associated to problem (55) can be solved more efficiently than the saddle-point system associated to problem (30). The discrete flux $\boldsymbol{\sigma}_{h}$ can then be recovered according to (52) by an element-byelement post-processing. 


\subsubsection{Link with the HHO method}

In [21], the authors study a HHO method based on the following bilinear form on $W_{h}^{k} \times W_{h}^{k}$, which only differs from $A$ (cf. (55)) in the choice of the stabilization term:

$$
A_{\mathrm{HHO}}\left(w_{h}, z_{h}\right)=\left(\boldsymbol{G}_{h}^{k} w_{h}, \boldsymbol{G}_{h}^{k} z_{h}\right)+j_{\mathrm{HHO}}\left(w_{h}, z_{h}\right),
$$

where, in comparison with (54), no link with a mixed hybrid method is used, but

$$
j_{\mathrm{HHO}}\left(w_{h}, z_{h}\right):=\sum_{T \in \mathcal{T}_{h}} \sum_{F \in \mathcal{F}_{T}}\left(\pi_{F}^{k}\left(\mathfrak{r}_{T}^{k} R_{W, T}^{k} w_{h}-\lambda_{F}\right), \pi_{F}^{k}\left(\mathfrak{r}_{T}^{k} R_{W, T}^{k} z_{h}-\mu_{F}\right)\right)_{F},
$$

and, for all $T \in \mathcal{T}_{h}$, the potential reconstruction operator $\mathfrak{r}_{T}^{k}: W_{T}^{k} \rightarrow \mathbb{P}_{d}^{k+1}(T)$ is such that, for all $z=\left(v_{T},\left(\mu_{F}\right)_{F \in \mathcal{F}_{T}}\right) \in W_{T}^{k}, \mathfrak{r}_{T}^{k} z=\left(r_{T}^{k} z-\pi_{T}^{k}\left(r_{T}^{k} z\right)\right)+v_{T}$ and $r_{T}^{k}$ is defined by (49). The stabilization bilinear forms $j$ (cf. (54)) and $j_{\mathrm{HHO}}$ are equivalent in that both of them (i) are polynomially consistent, i.e., they vanish when their $\operatorname{argument}$ is $I_{W, h}^{k} w$ with $w \in$ $\mathbb{P}_{d}^{k+1}\left(\mathcal{T}_{h}\right) \cap H_{0}^{1}(\Omega)$ and (ii) yield stability and continuity for $A$ in the form (56).

\section{Application to the Stokes problem}

In this section, we discuss a novel inf-sup stable discretization of the Stokes problem based on the hybridized MHO method. The continuous problem consists in seeking a velocity field $\boldsymbol{u}: \Omega \rightarrow \mathbb{R}^{d}$ and a pressure field $p: \Omega \rightarrow \mathbb{R}$ such that

$$
\begin{array}{rlrl}
-\triangle \boldsymbol{u}+\boldsymbol{\nabla} p & =\boldsymbol{f} & & \text { in } \Omega, \\
-\boldsymbol{\nabla} \cdot \boldsymbol{u} & =0 & & \text { in } \Omega, \\
\boldsymbol{u} & =\mathbf{0} & & \text { on } \partial \Omega, \\
(p, 1)_{\Omega}=0, &
\end{array}
$$

where $\boldsymbol{f}=\left(f_{i}\right)_{1 \leqslant i \leqslant d} \in L^{2}(\Omega)^{d}$ denotes the volumetric body force. Letting

$$
\boldsymbol{W}:=H_{0}^{1}(\Omega)^{d} \quad P:=L_{0}^{2}(\Omega),
$$

$\left(L_{0}^{2}(\Omega)\right.$ denotes the space of square-integrable functions with zero mean on $\Omega$ ), the weak formulation of (57) reads: Find $(\boldsymbol{u}, p) \in \boldsymbol{W} \times P$ such that

$$
\begin{aligned}
(\boldsymbol{\nabla} \boldsymbol{u}, \boldsymbol{\nabla} \boldsymbol{v})-(p, \boldsymbol{\nabla} \cdot \boldsymbol{v}) & =(\boldsymbol{f}, \boldsymbol{v}) & & \forall \boldsymbol{v} \in \boldsymbol{W}, \\
(\boldsymbol{\nabla} \cdot \boldsymbol{u}, q) & =0 & & \forall q \in P .
\end{aligned}
$$

The key idea is here to (i) discretize the diffusive term in the momentum conservation equation (59a) using the bilinear form $A$ defined by (53) for each component of the discrete velocity field (in view of the results in Section 3.3.4, one could alternatively use the bilinear form $A_{\mathrm{HHO}}$ defined by (3.3.4)); (ii) realize the velocity-pressure coupling by means of a discrete divergence operator $\mathcal{D}_{h}^{k}$ designed in the same spirit as $D_{h}^{k}$ (cf. (19)) and relying on the interpretation of the Lagrange multipliers as traces of the potential. 


\subsection{Degrees of freedom}

Recalling the definition (32) of $W_{T}^{k}$, we define, for all $T \in \mathcal{T}_{h}$, the local DOF space for the velocity as

$$
\boldsymbol{W}_{T}^{k}:=\left(W_{T}^{k}\right)^{d},
$$

while we seek the pressure in $\mathbb{P}_{d}^{k}(T)$. Correspondingly, the global DOF spaces for the velocity and pressure are given by

$$
\boldsymbol{W}_{h}^{k}:=\left(W_{h}^{k}\right)^{d}, \quad \mathcal{P}_{h}^{k}:=\mathbb{P}_{d}^{k}\left(\mathcal{T}_{h}\right) \cap L_{0}^{2}(\Omega) .
$$

We also define the local and global velocity interpolators $I_{\boldsymbol{W}, T}^{k}$ and $I_{\boldsymbol{W}, h}^{k}$ obtained applying component-wise the interpolators $I_{W, T}^{k}$ and $I_{W, h}^{k}$ defined by (39) and (40), respectively. Finally, for all $T \in \mathcal{T}_{h}$, we denote by $R_{\boldsymbol{W}, T}^{k}: \boldsymbol{W}_{h}^{k} \rightarrow \boldsymbol{W}_{T}^{k}$ the restriction operator that realizes the mapping between global and local velocity DOFs.

\section{$4.2 \quad$ Velocity-pressure coupling}

The velocity-pressure coupling is based on the local discrete divergence operator $\mathcal{D}_{T}^{k}: \boldsymbol{W}_{T}^{k} \rightarrow$ $\mathbb{P}_{d}^{k}(T)$ such that, for all $\boldsymbol{z}=\left(v_{T, i},\left(\mu_{F, i}\right)_{F \in \mathcal{F}_{T}}\right)_{1 \leqslant i \leqslant d} \in \boldsymbol{W}_{T}^{k}$,

$$
\left(\mathcal{D}_{T}^{k} \boldsymbol{z}, q\right)_{T}=\sum_{i=1}^{d}\left\{-\left(v_{T, i}, \partial_{i} q\right)_{T}+\sum_{F \in \mathcal{F}_{T}}\left(\mu_{F, i} n_{T F, i}, q\right)_{F}\right\} \quad \forall q \in \mathbb{P}_{d}^{k}(T),
$$

where $\partial_{i}$ denotes the partial derivative with respect to the $i$ th space variable. In the context of lowest-order methods for the Stokes problem, this formula for the divergence has been used, e.g., in $[8,22]$. In the higher-order case, it is essentially analogous (up to the choice of the discretization space for the velocity) to the one of [27, Section 4]. We record the following equivalence obtained integrating by parts the first term in (61):

$$
\left(\mathcal{D}_{T}^{k} \boldsymbol{z}, q\right)_{T}=\sum_{i=1}^{d}\left\{\left(\partial_{i} v_{T, i}, q\right)_{T}+\sum_{F \in \mathcal{F}_{T}}\left(\left(\mu_{F, i}-v_{T, i}\right) n_{T F, i}, q\right)_{F}\right\} \quad \forall q \in \mathbb{P}_{d}^{k}(T) .
$$

We also define the global discrete divergence operator $\mathcal{D}_{h}^{k}: \boldsymbol{W}_{h}^{k} \rightarrow \mathcal{P}_{h}^{k}$ such that, for all $\boldsymbol{z}_{h} \in \boldsymbol{W}_{h}^{k}$

$$
\left(\mathcal{D}_{h}^{k} \boldsymbol{z}_{h}, q_{h}\right)=\sum_{T \in \mathcal{T}_{h}}\left(\mathcal{D}_{T}^{k} R_{\boldsymbol{W}, T}^{k} \boldsymbol{z}_{h}, q_{h}\right)_{T} \quad \forall q_{h} \in \mathcal{P}_{h}^{k}
$$

The operator $\mathcal{D}_{h}^{k}$ defined by (63) can be regarded as the discrete counterpart of the divergence operator defined from $\boldsymbol{W}$ to $P$, cf. (58), as opposed to the operator $D_{h}^{k}$ defined by (19), which discretizes the divergence operator from $\boldsymbol{\Sigma}$ to $U$, cf. (7).

The following commuting property can be proved as the corresponding counterpart (20) for $D_{h}^{k}$ and is key to stability.

Proposition 5 (Commuting property for $\mathcal{D}_{h}^{k}$ ). The following commuting diagrams hold with $\boldsymbol{W}(T):=W(T)^{d}$ and $W(T)$ defined by $(38)$ : 

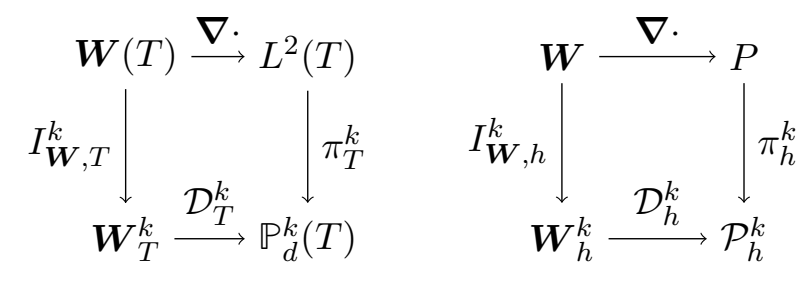

\subsection{Discrete problem}

The discretization of the viscous term in (59a) hinges on the bilinear form $\mathcal{A}$ on $\boldsymbol{W}_{h}^{k} \times \boldsymbol{W}_{h}^{k}$ such that, for all $\boldsymbol{w}_{h}=\left(w_{h, i}\right)_{1 \leqslant i \leqslant d}$ and $\boldsymbol{z}_{h}=\left(z_{h, i}\right)_{1 \leqslant i \leqslant d}$ in $\boldsymbol{W}_{h}^{k}$,

$$
\mathcal{A}\left(\boldsymbol{w}_{h}, \boldsymbol{z}_{h}\right):=\sum_{i=1}^{d} A\left(w_{h, i}, z_{h, i}\right),
$$

with bilinear form $A$ defined by (53). The coercivity and continuity of the bilinear form $\mathcal{A}$ follow from the corresponding properties (56) of the bilinear form $A$ :

$$
\eta\left\|\boldsymbol{z}_{h}\right\|_{1, h}^{2} \leqslant \mathcal{A}\left(\boldsymbol{z}_{h}, \boldsymbol{z}_{h}\right):=\left\|\boldsymbol{z}_{h}\right\|_{\mathcal{A}}^{2} \leqslant \eta^{-1}\left\|\boldsymbol{z}_{h}\right\|_{1, h}^{2},
$$

where $\left\|\boldsymbol{z}_{h}\right\|_{1, h}^{2}:=\sum_{i=1}^{d}\left\|z_{h, i}\right\|_{1, h}^{2}$ and the scalar version of the $\|\cdot\|_{1, h}$-norm is defined by (36). The source term in (59a) is discretized by means of the linear form $L$ on $\boldsymbol{W}_{h}^{k}$ such that, for all $\boldsymbol{z}_{h}=\left(v_{h, i}, \mu_{h, i}\right)_{1 \leqslant i \leqslant d}$,

$$
L\left(\boldsymbol{z}_{h}\right)=\sum_{i=1}^{d}\left(f_{i}, v_{h, i}\right) .
$$

The discretization of problem (59) reads: Find $\left(\boldsymbol{w}_{h}, p_{h}\right) \in \boldsymbol{W}_{h}^{k} \times \mathcal{P}_{h}^{k}$ such that

$$
\begin{aligned}
\mathcal{A}\left(\boldsymbol{w}_{h}, \boldsymbol{z}_{h}\right)-\left(p_{h}, \mathcal{D}_{h}^{k} \boldsymbol{z}_{h}\right) & =L\left(\boldsymbol{z}_{h}\right) & & \forall \boldsymbol{z}_{h} \in \boldsymbol{W}_{h}^{k}, \\
\left(\mathcal{D}_{h}^{k} \boldsymbol{w}_{h}, q_{h}\right) & =0 & & \forall q_{h} \in \mathcal{P}_{h}^{k} .
\end{aligned}
$$

The following result is a classical consequence of the commuting diagram property in Proposition 5 together with the surjectivity of the continuous divergence operator from $\boldsymbol{W}$ to $P$, cf. [10].

Lemma 6 (Well-posedness). There exists $\beta>0$ independent of $h$ such that, for all $q_{h} \in \mathcal{P}_{h}^{k}$, the following inf-sup condition holds:

$$
\beta\left\|q_{h}\right\| \leqslant \sup _{\boldsymbol{z}_{h} \in \boldsymbol{W}_{h}^{k} \backslash\{\boldsymbol{0}\}} \frac{\left(\mathcal{D}_{h}^{k} \boldsymbol{z}_{h}, q_{h}\right)}{\left\|\boldsymbol{z}_{h}\right\|_{1, h}} .
$$

Additionally, problem (67) is well-posed.

\subsection{Energy-norm convergence estimate}

Lemma 7 (Basic error estimate). Let $(\boldsymbol{u}, p) \in \boldsymbol{W} \times P$ denote the unique solution to (59), and let $\left(\widehat{\boldsymbol{w}}_{h}, \widehat{p}_{h}\right):=\left(I_{\boldsymbol{W}, h}^{k} \boldsymbol{u}, \pi_{h}^{k} p\right)$. Then, denoting by $\left(\boldsymbol{w}_{h}, p_{h}\right) \in \boldsymbol{W}_{h}^{k} \times \mathcal{P}_{h}^{k}$ the unique solution 


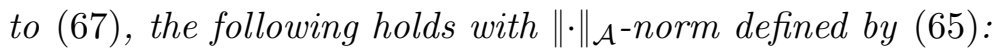

$$
\max \left(\frac{\beta \eta^{1 / 2}}{2}\left\|p_{h}-\widehat{p}_{h}\right\|,\left\|\boldsymbol{w}_{h}-\widehat{\boldsymbol{w}}_{h}\right\|_{\mathcal{A}}\right) \leqslant \sup _{\boldsymbol{z}_{h} \in \boldsymbol{W}_{h}^{k} \backslash\{\mathbf{0}\}} \frac{\mathcal{E}_{h}\left(\boldsymbol{z}_{h}\right)}{\left\|\boldsymbol{z}_{h}\right\|_{\mathcal{A}}},
$$

where the consistency error is such that $\mathcal{E}_{h}\left(\boldsymbol{z}_{h}\right)=L\left(\boldsymbol{z}_{h}\right)+\left(\widehat{p}_{h}, \mathcal{D}_{h}^{k} \boldsymbol{z}_{h}\right)-\mathcal{A}\left(\widehat{\boldsymbol{w}}_{h}, \boldsymbol{z}_{h}\right)$.

Proof. We denote by $\$$ the supremum in the right-hand side of (69). Observe that $\mathcal{D}_{h}^{k} \boldsymbol{w}_{h}=$ $\mathcal{D}_{h}^{k} \widehat{\boldsymbol{w}}_{h}=0$ as a consequence of $(67 \mathrm{~b})$ and the right commuting diagram in Proposition 5 together with $(57 \mathrm{~b})$, respectively. As a result, making $\boldsymbol{z}_{h}=\boldsymbol{w}_{h}-\widehat{\boldsymbol{w}}_{h}$ in (67a), and recalling the definition of the consistency error $\mathcal{E}_{h}$, one has

$$
\left\|\boldsymbol{w}_{h}-\widehat{\boldsymbol{w}}_{h}\right\|_{\mathcal{A}} \leqslant \$ .
$$

Let us now estimate the error on the pressure. Using (67a) together with the definition of the consistency error yields, for all $\boldsymbol{z}_{h} \in \boldsymbol{W}_{h}^{k}$,

$$
\left(p_{h}-\widehat{p}_{h}, \mathcal{D}_{h}^{k} \boldsymbol{z}_{h}\right)=\left(p_{h}, \mathcal{D}_{h}^{k} \boldsymbol{z}_{h}\right)-\left(\widehat{p}_{h}, \mathcal{D}_{h}^{k} \boldsymbol{z}_{h}\right)=\mathcal{A}\left(\boldsymbol{w}_{h}-\widehat{\boldsymbol{w}}_{h}, \boldsymbol{z}_{h}\right)-\mathcal{E}_{h}\left(\boldsymbol{z}_{h}\right) .
$$

Using the inf-sup condition (68) for $q_{h}=p_{h}-\widehat{p}_{h}$ together with (70), the Cauchy-Schwarz inequality, and the second inequality in (65), it is inferred that

$$
\beta \eta^{1 / 2}\left\|p_{h}-\widehat{p}_{h}\right\| \leqslant \sup _{\boldsymbol{z}_{h} \in \boldsymbol{W}_{h}^{k} \backslash\{\mathbf{0}\}} \frac{\left(p_{h}-\widehat{p}_{h}, \mathcal{D}_{h}^{k} \boldsymbol{z}_{h}\right)}{\eta^{-1 / 2}\left\|\boldsymbol{z}_{h}\right\|_{1, h}} \leqslant\left\|\boldsymbol{w}_{h}-\widehat{\boldsymbol{w}}_{h}\right\|_{\mathcal{A}}+\$ \leqslant 2 \$ .
$$

The estimate (69) is an immediate consequence of (70)-(71).

Theorem 8 (Convergence rate for the energy-norm of the error). Under the assumptions of Lemma 7 , and assuming the additional regularity $\boldsymbol{u} \in H^{k+2}\left(\mathcal{T}_{h}\right)^{d}$ and $p \in H^{k+1}\left(\mathcal{T}_{h}\right)$, the following holds:

$$
\max \left(\frac{\beta \eta^{1 / 2}}{2}\left\|p_{h}-\widehat{p}_{h}\right\|,\left\|\boldsymbol{w}_{h}-\widehat{\boldsymbol{w}}_{h}\right\|_{\mathcal{A}}\right) \leqslant C h^{k+1}\left(\|\boldsymbol{u}\|_{H^{k+2}\left(\mathcal{T}_{h}\right)^{d}}+\|p\|_{H^{k+1}\left(\mathcal{T}_{h}\right)}\right),
$$

with $C>0$ independent of $h$.

Proof. For a given $\boldsymbol{z}_{h}=\left(\left(v_{T, i}\right)_{T \in \mathcal{T}_{h}},\left(\mu_{F, i}\right)_{F \in \mathcal{F}_{h}}\right)_{1 \leqslant i \leqslant d} \in \boldsymbol{W}_{h}^{k}$, we introduce the vector-valued polynomial functions $\boldsymbol{v}_{T}:=\left(v_{T, i}\right)_{1 \leqslant i \leqslant d}$ for all $T \in \mathcal{T}_{h}$ and $\boldsymbol{\mu}_{F}:=\left(\mu_{F, i}\right)_{1 \leqslant i \leqslant d}$ for all $F \in \mathcal{F}_{h}$. We also introduce $\breve{\boldsymbol{u}}_{h}=\left(\breve{u}_{h, i}\right)_{1 \leqslant i \leqslant d}$ where, for all $1 \leqslant i \leqslant d, \breve{u}_{h, i}:=r_{T}^{k} \widehat{w}_{h, i}$ and $r_{T}^{k}$ is the potential reconstruction operator defined by (49). Using the fact that $\boldsymbol{f}=-\Delta \boldsymbol{u}+\boldsymbol{\nabla} p$ a.e. in $\Omega$, recalling the definitions (64) of the bilinear form $\mathcal{A}$ and (53) of the bilinear form $A$ together with (62), and performing an element-by-element integration by parts on the linear form $L$ defined by (66), we decompose the consistency error as follows:

$$
\begin{aligned}
\mathcal{E}_{h}\left(\boldsymbol{z}_{h}\right)= & \sum_{T \in \mathcal{T}_{h}}\left\{\left(\boldsymbol{\nabla}\left(\boldsymbol{u}-\breve{\boldsymbol{u}}_{h}\right), \boldsymbol{\nabla} \boldsymbol{v}_{T}\right)_{T}+\sum_{F \in \mathcal{F}_{T}}\left(\boldsymbol{\nabla}\left(\boldsymbol{u}-\breve{\boldsymbol{u}}_{h \mid T}\right) \boldsymbol{n}_{T F}, \boldsymbol{\mu}_{F}-\boldsymbol{v}_{T}\right)_{F}\right\} \\
& +\sum_{T \in \mathcal{T}_{h}}\left\{\left(\widehat{p}_{h}-p, \boldsymbol{\nabla} \cdot \boldsymbol{v}_{T}\right)_{T}+\sum_{F \in \mathcal{F}_{T}}\left(\widehat{p}_{h}-p,\left(\boldsymbol{\mu}_{F}-\boldsymbol{v}_{T}\right) \cdot \boldsymbol{n}_{T F}\right)_{F}\right\}+\sum_{i=1}^{d} j\left(\widehat{w}_{h, i}, z_{h, i}\right),
\end{aligned}
$$


where we have used continuity of the normal momentum flux across interfaces as well as the fact that the homogeneous Dirichlet boundary condition is embedded in $\boldsymbol{W}_{h}^{k}$ (cf. (60) and (32)) to introduce the term $\sum_{T \in \mathcal{T}_{h}} \sum_{F \in \mathcal{F}_{T}}\left(\left(\boldsymbol{\nabla} \boldsymbol{u}-p \mathbf{I}_{d}\right) \boldsymbol{n}_{T F}, \boldsymbol{\mu}_{F}\right)_{F}$.

Denote by $\mathfrak{T}_{1}, \mathfrak{T}_{2}$, and $\mathfrak{T}_{3}$ the terms in the right-hand side of (73). Multiple uses of the Cauchy-Schwarz inequality followed by the approximation properties (50) of $r_{T}^{k}$ and (4) of the $L^{2}$-orthogonal projector yield

$$
\begin{aligned}
\left|\mathfrak{T}_{1}\right|+\left|\mathfrak{T}_{2}\right| & \leqslant h^{k+1}\left(\|\boldsymbol{u}\|_{H^{k+2}\left(\mathcal{T}_{h}\right)^{d}}+\|p\|_{H^{k+1}\left(\mathcal{T}_{h}\right)}\right)\left\|\boldsymbol{z}_{h}\right\|_{1, h} \\
& \lesssim h^{k+1}\left(\|\boldsymbol{u}\|_{H^{k+2}\left(\mathcal{T}_{h}\right)^{d}}+\|p\|_{H^{k+1}\left(\mathcal{T}_{h}\right)}\right)\left\|\boldsymbol{z}_{h}\right\|_{\mathcal{A}},
\end{aligned}
$$

where to conclude we have used the first inequality in (44). Let us now turn to the estimate of the stabilization term $\mathfrak{T}_{3}$. Recall that $\widehat{\boldsymbol{w}}_{h}=\left(\widehat{w}_{h, i}\right)_{1 \leqslant i \leqslant d}$ with $\widehat{w}_{h, i}=\left(\left(\widehat{u}_{T, i}\right)_{T \in \mathcal{T}_{h}},\left(\widehat{\lambda}_{F, i}\right)_{F \in \mathcal{F}_{h}}\right)$ for all $1 \leqslant i \leqslant d$. Using the definitions (64) of $\mathcal{A}$ and (53) of $A$, and letting $\boldsymbol{\tau}_{h, i}=\boldsymbol{\varsigma}_{h}^{k} z_{h, i}$ for all $1 \leqslant i \leqslant d$, it is inferred

$$
\begin{aligned}
\mathfrak{T}_{3} & =\sum_{i=1}^{d}\left\{H\left(\boldsymbol{\varsigma}_{h}^{k} \widehat{w}_{h, i}, \boldsymbol{\tau}_{h, i}\right)-\left(\boldsymbol{G}_{h}^{k} \widehat{w}_{h, i}, \boldsymbol{G}_{h}^{k} z_{h, i}\right)\right\} \\
& =\sum_{i=1}^{d} \sum_{T \in \mathcal{T}_{h}}\left\{\left(\boldsymbol{\nabla} \widehat{u}_{T, i}, \boldsymbol{\tau}_{T, i}\right)_{T}+\sum_{F \in \mathcal{F}_{T}}\left(\widehat{\lambda}_{F, i}-\widehat{u}_{T, i}, \tau_{T F, i}\right)_{F}-\left(\boldsymbol{\nabla} \breve{u}_{T, i}, \mathfrak{C}_{T}^{k} R_{\boldsymbol{\Sigma}, T}^{k} \boldsymbol{\tau}_{h, i}\right)_{T}\right\} \\
& =\sum_{i=1}^{d} \sum_{T \in \mathcal{T}_{h}}\left\{\left(\boldsymbol{\nabla}\left(\widehat{u}_{T, i}-\pi_{T}^{k} \breve{u}_{T, i}\right), \boldsymbol{\tau}_{T, i}\right)_{T}+\sum_{F \in \mathcal{F}_{T}}\left(\widehat{\lambda}_{F, i}-\pi_{F}^{k} \breve{u}_{T, i}-\widehat{u}_{T, i}+\pi_{T}^{k} \breve{u}_{T, i}, \tau_{T F, i}\right)_{F}\right\} \\
& =\sum_{i=1}^{d} \sum_{T \in \mathcal{T}_{h}}\left\{\left(\boldsymbol{\nabla} \pi_{T}^{k}\left(u_{i}-\breve{u}_{T, i}\right), \boldsymbol{\tau}_{T, i}\right)_{T}+\sum_{F \in \mathcal{F}_{T}}\left(\pi_{F}^{k}\left(u_{i}-\breve{u}_{T, i}\right)+\pi_{T}^{k}\left(\breve{u}_{T, i}-u_{i}\right), \tau_{T F, i}\right)_{F}\right\},
\end{aligned}
$$

where we have used (53) and (41b) together with $G_{T}^{k} R_{W, T}^{k} \widehat{w}_{h, i}=\nabla \breve{u}_{T, i}$ for all $1 \leqslant i \leqslant d$ to pass to the second line, (21b) to pass to the third, and (40) to conclude. Using the Cauchy-Schwarz, discrete inverse (3) and trace (2) inequalities for the terms involving $\pi_{T}^{k}$, and recalling that, by definition, $\boldsymbol{\tau}_{h, i}=\boldsymbol{\varsigma}_{h}^{k} z_{h, i}$ for all $1 \leqslant i \leqslant d$, it is inferred,

$$
\begin{aligned}
\left|\mathfrak{T}_{3}\right| & \lesssim\left\{\sum_{T \in \mathcal{T}_{h}}\left[h_{T}^{-2}\left\|\pi_{T}^{k}\left(\boldsymbol{u}-\check{\boldsymbol{u}}_{T}\right)\right\|_{T}^{2}+\sum_{F \in \mathcal{F}_{T}} h_{F}^{-1}\left\|\pi_{F}^{k}\left(\boldsymbol{u}-\check{\boldsymbol{u}}_{T}\right)\right\|_{F}^{2}\right]\right\}^{1 / 2} \times\left\{\sum_{i=1}^{d}\left\|\boldsymbol{\varsigma}_{h}^{k} z_{h, i}\right\|^{2}\right\}^{1 / 2} \\
& \lesssim h^{k+1}\|\boldsymbol{u}\|_{H^{k+2}\left(\mathcal{T}_{h}\right)^{d}}\left\|\boldsymbol{z}_{h}\right\|_{\mathcal{A}},
\end{aligned}
$$

where we have concluded using the fact that $\pi_{T}^{k}$ and $\pi_{F}^{k}$ are bounded operators as projectors, the approximation properties (50) of the potential reconstruction, and recalling (65) after observing that $\sum_{i=1}^{d}\left\|z_{h, i}\right\|_{A}^{2}=\left\|\boldsymbol{z}_{h}\right\|_{\mathcal{A}}^{2}$. Finally, to prove the estimate (72), use (74)-(75) to bound the right-hand side of (73) and plug the resulting bound into (69).

\section{5 $\quad L^{2}$-norm convergence estimate for the velocity}

The estimate for the $L^{2}$-norm of the velocity can be refined assuming further regularity for problem (57). We assume in this section that Cattabriga's regularity holds (cf. Cattabriga [13] 
and also Amrouche and Girault [1]) in the following form: There is $C_{\text {Cat }}$, only depending on $\Omega$ such that, for all $\boldsymbol{g} \in L^{2}(\Omega)^{d}$, denoting by $(\boldsymbol{z}, r) \in \boldsymbol{W} \times P$ the unique solution to

$$
\begin{aligned}
(\boldsymbol{\nabla} \boldsymbol{z}, \boldsymbol{\nabla} \boldsymbol{v})-(r, \boldsymbol{\nabla} \cdot \boldsymbol{v}) & =(\boldsymbol{g}, \boldsymbol{v}) & & \forall \boldsymbol{v} \in \boldsymbol{W}, \\
(\boldsymbol{\nabla} \cdot \boldsymbol{z}, q) & =0 & & \forall q \in P,
\end{aligned}
$$

it holds that

$$
\|\boldsymbol{z}\|_{H^{2}(\Omega)^{d}}+\|r\|_{H^{1}(\Omega)} \leqslant C_{\text {Cat }}\|\boldsymbol{g}\| .
$$

The following result shows that supercloseness holds for the velocity element DOFs, which converge with order $(k+2)$ to the $L^{2}$-orthogonal projection of the velocity on the broken polynomial space $\mathbb{P}_{d}^{k}\left(\mathcal{T}_{h}\right)^{d}$.

Theorem 9 (Convergence rate for the $L^{2}$-norm of the error on the velocity). Under the assumptions of Theorem 8, and assuming that Cattabriga's regularity (77) holds, there exists a real number $C>0$ independent of $h$ such that, if $k \geqslant 1$,

$$
\left\|\boldsymbol{u}_{h}-\widehat{\boldsymbol{u}}_{h}\right\| \leqslant C h^{k+2}\left(\|\boldsymbol{u}\|_{H^{k+2}\left(\mathcal{T}_{h}\right)^{d}}+\|p\|_{H^{k+1}\left(\mathcal{T}_{h}\right)}\right),
$$

and, for $k=0$, further assuming that $\boldsymbol{f} \in H^{1}(\Omega)^{d}$,

$$
\left\|\boldsymbol{u}_{h}-\widehat{\boldsymbol{u}}_{h}\right\| \leqslant C h^{2}\|\boldsymbol{f}\|_{H^{1}(\Omega)^{d}},
$$

where $\boldsymbol{u}_{h}, \widehat{\boldsymbol{u}}_{h} \in \mathbb{P}_{d}^{k}\left(\mathcal{T}_{h}\right)^{d}$ are obtained from element unknowns setting, for all $T \in \mathcal{T}_{h}$,

$$
\boldsymbol{u}_{h \mid T}=\left(u_{T, i}\right)_{1 \leqslant i \leqslant d}, \quad \widehat{\boldsymbol{u}}_{h \mid T}=\left(\widehat{u}_{T, i}\right)_{1 \leqslant i \leqslant d} .
$$

Proof. Let $\boldsymbol{z}$ solve (76) with $\boldsymbol{g}=\widehat{\boldsymbol{u}}_{h}-\boldsymbol{u}_{h}$, set $\widehat{\boldsymbol{z}}_{h}:=I_{\boldsymbol{W}, h}^{k} \boldsymbol{z}$, and define the error on the velocity

$$
\boldsymbol{e}_{h}:=\widehat{\boldsymbol{w}}_{h}-\boldsymbol{w}_{h}=\left(\left(\epsilon_{T, i}\right)_{T \in \mathcal{T}_{h}},\left(\rho_{F, i}\right)_{F \in \mathcal{F}_{h}}\right)_{1 \leqslant i \leqslant d} \in \boldsymbol{W}_{h}^{k} .
$$

We also introduce the following vector-valued quantities obtained from the element and face DOFs of $\boldsymbol{e}_{h}$, respectively:

$$
\boldsymbol{\epsilon}_{T}=\left(\epsilon_{T, i}\right)_{1 \leqslant i \leqslant d} \quad \forall T \in \mathcal{T}_{h} \quad \text { and } \quad \boldsymbol{\rho}_{F}=\left(\rho_{F, i}\right)_{1 \leqslant i \leqslant d} \quad \forall F \in \mathcal{F}_{h} .
$$

Using the fact that $-\triangle \boldsymbol{z}+\boldsymbol{\nabla} r=\widehat{\boldsymbol{u}}_{h}-\boldsymbol{u}_{h}=\boldsymbol{\epsilon}_{h}$ a.e. in $\Omega$, it holds for all $T \in \mathcal{T}_{h}$, integrating by parts and exploiting the flux continuity and the fact that $\boldsymbol{\rho}_{F} \equiv \mathbf{0}$ for all $F \in \mathcal{F}_{h}^{\mathrm{b}}$ to insert the term $0=\sum_{T \in \mathcal{T}_{h}} \sum_{F \in \mathcal{F}_{T}}\left(\boldsymbol{\rho}_{F},\left(\boldsymbol{\nabla} \boldsymbol{z}-r \mathbf{I}_{d}\right) \boldsymbol{n}_{T F}\right)_{F}$,

$$
\left\|\boldsymbol{u}_{h}-\widehat{\boldsymbol{u}}_{h}\right\|_{T}^{2}=\left(\boldsymbol{\nabla} \boldsymbol{\epsilon}_{T}, \boldsymbol{\nabla} \boldsymbol{z}-r \mathbf{I}_{d}\right)_{T}+\sum_{T \in \mathcal{T}_{h}} \sum_{F \in \mathcal{F}_{T}}\left(\left(\boldsymbol{\rho}_{F}-\boldsymbol{\epsilon}_{T}\right),\left(\boldsymbol{\nabla} \boldsymbol{z}-r \mathbf{I}_{d}\right) \boldsymbol{n}_{T F}\right)_{F} .
$$

Adding to the above expression the quantity (cf. (67a))

$$
0=\mathcal{A}\left(\boldsymbol{w}_{h}, \hat{\boldsymbol{z}}_{h}\right)-\left(p_{h}, \mathcal{D}_{h}^{k} \widehat{\boldsymbol{z}}_{h}\right)-\sum_{T \in \mathcal{T}_{h}}\left(\boldsymbol{f}, \pi_{T}^{k} \boldsymbol{z}\right)_{T}=\mathcal{A}\left(\widehat{\boldsymbol{w}}_{h}, \widehat{\boldsymbol{z}}_{h}\right)-\mathcal{A}\left(\boldsymbol{e}_{h}, \hat{\boldsymbol{z}}_{h}\right)-\sum_{T \in \mathcal{T}_{h}}\left(\boldsymbol{f}, \pi_{T}^{k} \boldsymbol{z}\right)_{T},
$$

where we have used Proposition 5 together with $(76 \mathrm{~b})$ to infer $\mathcal{D}_{h}^{k} \widehat{\boldsymbol{z}}_{h}=\pi_{h}^{k}(\boldsymbol{\nabla} \cdot \boldsymbol{z})=0$, we have

$$
\left\|\boldsymbol{u}_{h}-\widehat{\boldsymbol{u}}_{h}\right\|^{2}=\mathfrak{T}_{1}+\mathfrak{T}_{2}+\mathfrak{T}_{3},
$$


with

$$
\begin{aligned}
& \mathfrak{T}_{1}:=\sum_{T \in \mathcal{T}_{h}}\left\{\left(\boldsymbol{\nabla} \boldsymbol{\epsilon}_{T}, \boldsymbol{\nabla} \boldsymbol{z}\right)_{T}+\sum_{F \in \mathcal{F}_{T}}\left(\boldsymbol{\rho}_{F}-\boldsymbol{\epsilon}_{T}, \boldsymbol{\nabla} \boldsymbol{z} \boldsymbol{n}_{T F}\right)_{F}\right\}-\mathcal{A}\left(\boldsymbol{e}_{h}, \widehat{\boldsymbol{z}}_{h}\right), \\
& \mathfrak{T}_{2}:=-\sum_{T \in \mathcal{T}_{h}}\left\{\left(\boldsymbol{\nabla} \cdot \boldsymbol{\epsilon}_{T}, r\right)_{T}+\sum_{F \in \mathcal{F}_{T}}\left(\left(\boldsymbol{\rho}_{F}-\boldsymbol{\epsilon}_{T}\right) \cdot \boldsymbol{n}_{T F}, r\right)_{F}\right\} \\
& \mathfrak{T}_{3}:=\mathcal{A}\left(\widehat{\boldsymbol{w}}_{h}, \widehat{\boldsymbol{z}}_{h}\right)-\sum_{T \in \mathcal{T}_{h}}\left(\boldsymbol{f}, \pi_{T}^{k} \boldsymbol{z}\right)_{T} .
\end{aligned}
$$

To bound $\mathfrak{T}_{1}$ we recall the definitions (64) of $\mathcal{A}$ and (53) of $A$, and observe that, with $\boldsymbol{\delta}_{T}:=\left(z_{i \mid T}-r_{T}^{k} I_{W, T}^{k} z_{i \mid T}\right)_{1 \leqslant i \leqslant d}$,

$$
\mathfrak{T}_{1}=\sum_{T \in \mathcal{T}_{h}}\left\{\left(\boldsymbol{\nabla} \boldsymbol{\epsilon}_{T}, \boldsymbol{\nabla} \boldsymbol{\delta}_{T}\right)_{T}+\sum_{F \in \mathcal{F}_{T}}\left(\boldsymbol{\rho}_{F}-\boldsymbol{\epsilon}_{T}, \boldsymbol{\nabla} \boldsymbol{\delta}_{T} \boldsymbol{n}_{T F}\right)_{F}\right\}+\mathcal{J}\left(\boldsymbol{e}_{h}, \hat{\boldsymbol{z}}_{h}\right),
$$

where, for the sake of brevity, we have introduced the bilinear form $\mathcal{J}\left(\boldsymbol{w}_{h}, \boldsymbol{v}_{h}\right):=\sum_{i=1}^{d} J\left(w_{h, i}, v_{h, i}\right)$. Hence, we infer

$$
\begin{aligned}
\left|\mathfrak{T}_{1}\right| & \leqslant\left\{\left\|\boldsymbol{e}_{h}\right\|_{1, h}^{2}+\mathcal{J}\left(\boldsymbol{e}_{h}, \boldsymbol{e}_{h}\right)\right\}^{1 / 2} \times\left\{\sum_{T \in \mathcal{T}_{h}}\left[\left\|\boldsymbol{\delta}_{T}\right\|_{T}^{2}+\left\|\boldsymbol{\delta}_{T}\right\|_{\partial T}^{2}\right]+\mathcal{J}\left(\widehat{\boldsymbol{z}}_{h}, \widehat{\boldsymbol{z}}_{h}\right)\right\}^{1 / 2} \\
& \leqslant h^{k+1}\left(\|\boldsymbol{u}\|_{H^{k+2}\left(\mathcal{T}_{h}\right)^{d}}+\|p\|_{H^{k+1}\left(\mathcal{T}_{h}\right)}\right) h\|\boldsymbol{z}\|_{H^{2}(\Omega)^{d}} \\
& \lesssim h^{k+2}\left(\|\boldsymbol{u}\|_{H^{k+2}\left(\mathcal{T}_{h}\right)^{d}}+\|p\|_{H^{k+1}\left(\mathcal{T}_{h}\right)}\right)\left\|\widehat{\boldsymbol{u}}_{h}-\boldsymbol{u}_{h}\right\|
\end{aligned}
$$

where we have used the Cauchy-Schwarz inequality followed by the energy estimate (72) for the first factor, while, for the second factor, we have estimated $\boldsymbol{\delta}_{T}$ using (50), $\mathcal{J}\left(\widehat{\boldsymbol{z}}_{h}, \widehat{\boldsymbol{z}}_{h}\right)$ as the term $\mathfrak{T}_{3}$ in the proof of Theorem 8, and we have used Cattabriga's regularity (77) for $\boldsymbol{z}$ to conclude.

To estimate $\mathfrak{T}_{2}$, we observe that $\mathcal{D}_{h}^{k} \boldsymbol{e}_{h}=\mathcal{D}_{h}^{k} \widehat{\boldsymbol{w}}_{h}-\mathcal{D}_{h}^{k} \boldsymbol{w}_{h}=0$ owing to Proposition 5 together with (59b) and (67b), hence, letting $r_{h}:=\pi_{h}^{k} r$ and using (62) with $\boldsymbol{z}=R_{\boldsymbol{W}, T}^{k} \boldsymbol{e}_{h}$ and $q=r_{T}$, we infer

$$
0=\left(\mathcal{D}_{h}^{k} \boldsymbol{e}_{h}, r_{h}\right)=\sum_{T \in \mathcal{T}_{h}}\left\{\left(\boldsymbol{\nabla} \cdot \boldsymbol{\epsilon}_{T}, r_{T}\right)_{T}+\sum_{F \in \mathcal{F}_{T}}\left(\left(\boldsymbol{\rho}_{F}-\boldsymbol{\epsilon}_{T}\right) \cdot \boldsymbol{n}_{T F}, r_{T}\right)_{F}\right\} .
$$

Subtracting the above expression from $\mathfrak{T}_{2}$, and using the Cauchy-Schwarz inequality together with the bound (1) on $\mathfrak{N}_{\partial}$, it is inferred

$\left|\mathfrak{T}_{2}\right| \lesssim\left\|\boldsymbol{e}_{h}\right\|_{1, h}\left\{\sum_{T \in \mathcal{T}_{h}}\left[\left\|r-r_{T}\right\|_{T}^{2}+\left\|r-r_{T}\right\|_{\partial T}^{2}\right]\right\}^{1 / 2} \lesssim h^{k+2}\left(\|\boldsymbol{u}\|_{H^{k+2}\left(\mathcal{T}_{h}\right)^{d}}+\|p\|_{H^{k+1}\left(\mathcal{T}_{h}\right)}\right)\|r\|_{H^{1}(\Omega)}$,

where we have used the first inequality in (65) together with the energy estimate (72) for the first factor and the approximation properties (4) of $\pi_{h}^{k}$ for the second.

Let us now estimate $\mathfrak{T}_{3}$. For all $T \in \mathcal{T}_{h}$, we have $\left(\boldsymbol{f}, \pi_{T}^{k} \boldsymbol{z}\right)_{T}=\left(\pi_{T}^{k} \boldsymbol{f}, \boldsymbol{z}\right)_{T}$. Moreover, since $(\boldsymbol{f}, \boldsymbol{z})=\left(\boldsymbol{\nabla} \boldsymbol{u}-p \mathbf{I}_{d}, \boldsymbol{\nabla} \boldsymbol{z}\right)$ and, owing to $(63),\left(\pi_{h}^{k} p, \mathcal{D}_{h}^{k} \widehat{\boldsymbol{z}}_{h}\right)=\left(p, \pi_{h}^{k}(\boldsymbol{\nabla} \cdot \boldsymbol{z})\right)=\left(\pi_{h}^{k} p, \boldsymbol{\nabla} \cdot \boldsymbol{z}\right)$, we 
infer

$$
\begin{aligned}
\mathfrak{T}_{3} & =\left(\boldsymbol{f}-\pi_{h}^{k} \boldsymbol{f}, \boldsymbol{z}\right) \\
& -\sum_{T \in \mathcal{T}_{h}}\left\{\sum_{i=1}^{d}\left[\left(\boldsymbol{\nabla} u_{i}, \boldsymbol{\nabla} z_{i}\right)_{T}-\left(\boldsymbol{G}_{T}^{k} I_{W, T}^{k} u_{i}, \boldsymbol{G}_{T}^{k} I_{W, T}^{k} z_{i}\right)\right]-\left(p-\pi_{h}^{k} p, \boldsymbol{\nabla} \cdot \boldsymbol{z}\right)\right\}+\mathcal{J}\left(\widehat{\boldsymbol{w}}_{h}, \widehat{\boldsymbol{z}}_{h}\right) .
\end{aligned}
$$

Denote by $\mathfrak{T}_{3,1}, \mathfrak{T}_{3,2}, \mathfrak{T}_{3,3}$ the addends in the right-hand side. If $k \geqslant 1$, we can write

$$
\left(\boldsymbol{f}-\pi_{h}^{k} \boldsymbol{f}, \boldsymbol{z}\right)=\left(\boldsymbol{f}-\pi_{h}^{k} \boldsymbol{f}, \boldsymbol{z}-\pi_{h}^{1} \boldsymbol{z}\right),
$$

hence

$$
\left|\mathfrak{T}_{3,1}\right| \lesssim h^{k}\|\boldsymbol{f}\|_{H^{k}(\Omega)^{d}} h^{2}\|\boldsymbol{z}\|_{H^{2}(\Omega)^{d}} \lesssim h^{k+2}\|\boldsymbol{f}\|_{H^{k}(\Omega)^{d}}\left\|\widehat{\boldsymbol{u}}_{h}-\boldsymbol{u}_{h}\right\|_{H^{2}(\Omega)^{d}} .
$$

On the other hand, for $k=0$, we write $\left(\boldsymbol{f}-\pi_{h}^{0} \boldsymbol{f}, \boldsymbol{z}-\pi_{h}^{0} \boldsymbol{z}\right)$ so that

$$
\left|\mathfrak{T}_{3,1}\right| \lesssim h\|\boldsymbol{f}\|_{H^{1}(\Omega)^{d}} h\|\boldsymbol{z}\|_{H^{1}(\Omega)^{d}} \lesssim h^{2}\|\boldsymbol{f}\|_{H^{1}(\Omega)^{d}}\left\|\widehat{\boldsymbol{u}}_{h}-\boldsymbol{u}_{h}\right\| .
$$

To estimate $\mathfrak{T}_{3,2}$ we use the orthogonality property (47) to infer

$$
\mathfrak{T}_{3,2}=\sum_{T \in \mathcal{T}_{h}} \sum_{i=1}^{d}\left[\left(\nabla u_{i}-\boldsymbol{G}_{T}^{k} I_{W, T}^{k} u_{i}, \nabla z_{i}-\boldsymbol{G}_{T}^{k} I_{W, T}^{k} z_{i}\right)\right],
$$

hence, recalling (50) and using Cattabriga's regularity (77) for $\boldsymbol{z}$, it is inferred $\left|\mathfrak{T}_{3,2}\right| \lesssim$ $h^{k+2}\|\boldsymbol{u}\|_{H^{k+2}(\Omega) d}\left\|\widehat{\boldsymbol{u}}_{h}-\boldsymbol{u}_{h}\right\|$. Finally, using the Cauchy-Schwarz inequality, proceeding as for the estimate of $\mathfrak{T}_{3}$ in the proof of Theorem 8, and recalling again (77), it is inferred

$\left|\mathfrak{T}_{3,3}\right| \leqslant \mathcal{J}\left(\widehat{\boldsymbol{w}}_{h}, \widehat{\boldsymbol{w}}_{h}\right)^{1 / 2} \mathcal{J}\left(\widehat{\boldsymbol{z}}_{h}, \widehat{\boldsymbol{z}}_{h}\right)^{1 / 2} \lesssim h^{k+1}\|\boldsymbol{u}\|_{H^{k+2}(\Omega)^{d}} h\|\boldsymbol{z}\|_{H^{2}(\Omega)^{d}} \lesssim h^{k+2}\|\boldsymbol{u}\|_{H^{k+2}(\Omega)^{d}}\left\|\widehat{\boldsymbol{u}}_{h}-\boldsymbol{u}_{h}\right\|$.

When $k=0$, using Cattabriga's regularity for $\boldsymbol{u}$ leads to the estimate $\left|\mathfrak{T}_{3,2}\right|+\left|\mathfrak{T}_{3,3}\right| \lesssim$ $h^{2}\|\boldsymbol{f}\|_{H^{1}(\Omega)^{d}}\left\|\widehat{\boldsymbol{u}}_{h}-\boldsymbol{u}_{h}\right\|$. Collecting the above estimates, it is finally inferred that

$$
\left|\mathfrak{T}_{3}\right| \lesssim h^{k+2}\|\boldsymbol{u}\|_{H^{k+2}(\Omega) d}\left\|\widehat{\boldsymbol{u}}_{h}-\boldsymbol{u}_{h}\right\|,
$$

which, used in conjunction with (81) and (82) (and Cattabriga's regularity for $(\boldsymbol{u}, p)$ when $k=0)$ to bound the right-hand side of (80) gives the desired result.

To close this section, we exhibit a discrete velocity reconstruction that converges with order $(k+2)$ to the exact velocity $\boldsymbol{u}$. Let, for all $T \in \mathcal{T}_{h}, \boldsymbol{r}_{T}^{k}: \boldsymbol{W}_{T}^{k} \rightarrow \mathbb{P}_{d}^{k+1}(T)^{d}$ denote the velocity reconstruction operator such that, for all $\boldsymbol{w} \in \boldsymbol{W}_{T}^{k}$,

$$
\boldsymbol{r}_{T}^{k} \boldsymbol{w}=\left(r_{T}^{k} w_{i}\right)_{1 \leqslant i \leqslant d}
$$

with $r_{T}^{k}$ defined by (49), and define its global counterpart $\boldsymbol{r}_{h}^{k}: \boldsymbol{W}_{h}^{k} \rightarrow \mathbb{P}_{d}^{k+1}\left(\mathcal{T}_{h}\right)^{d}$ such that, for all $\boldsymbol{v}_{h} \in \boldsymbol{W}_{h}^{k}$,

$$
\boldsymbol{r}_{h}^{k} \boldsymbol{v}_{h \mid T}=\boldsymbol{r}_{T}^{k} R_{\boldsymbol{W}, T}^{k} \boldsymbol{v}_{h}, \quad \forall T \in \mathcal{T}_{h} .
$$

Corollary 10 (Convergence of $\boldsymbol{r}_{h}^{k} \boldsymbol{w}_{h}$ ). Using the notation of Theorem 8, and under the assumptions of Theorem 9, there is a real number $C$ independent of $h$ such that

$$
\left\|\boldsymbol{u}-\boldsymbol{r}_{h}^{k} \boldsymbol{w}_{h}\right\| \leqslant C h^{k+2}\left(\|\boldsymbol{u}\|_{H^{k+2}\left(\mathcal{T}_{h}\right)^{d}}+\|p\|_{H^{k+1}\left(\mathcal{T}_{h}\right)}\right) .
$$



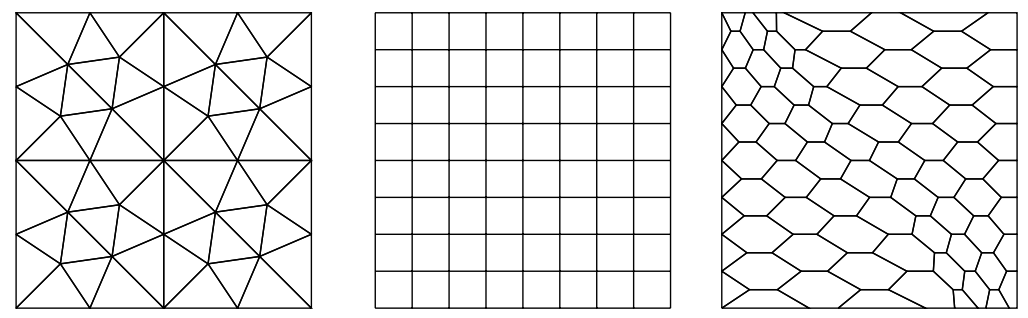

Figure 1: Triangular (Tria), Cartesian (Cart) and hexagonal (Hex) mesh families for the numerical example of Section 4.6

Proof. Recalling that $\widehat{\boldsymbol{w}}_{h}=I_{\boldsymbol{W}, h}^{k} \boldsymbol{u}$, and using the triangular inequality, one has

$$
\left\|\boldsymbol{u}-\boldsymbol{r}_{h}^{k} \boldsymbol{w}_{h}\right\| \leqslant\left\|\boldsymbol{u}-\boldsymbol{r}_{h}^{k} \widehat{\boldsymbol{w}}_{h}\right\|+\left\|\boldsymbol{r}_{h}^{k}\left(\widehat{\boldsymbol{w}}_{h}-\boldsymbol{w}_{h}\right)\right\|:=\mathfrak{T}_{1}+\mathfrak{T}_{2} .
$$

As a result of (50) it is readily inferred $\left|\mathfrak{T}_{1}\right| \lesssim h^{k+2}\|\boldsymbol{u}\|_{H^{k+2}\left(\mathcal{T}_{h}\right)^{d}}$. Additionally, one has,

$$
\mathfrak{T}_{2}=\sum_{T \in \mathcal{T}_{h}}\left\|\boldsymbol{r}_{T}^{k} R_{\boldsymbol{W}, T}^{k}\left(\widehat{\boldsymbol{w}}_{h}-\boldsymbol{w}_{h}\right)\right\|_{T}^{2} \lesssim \sum_{T \in \mathcal{T}_{h}}\left\{h_{T}^{2}\left\|\boldsymbol{\nabla} \boldsymbol{r}_{T}^{k} R_{\boldsymbol{W}, T}^{k}\left(\widehat{\boldsymbol{w}}_{h}-\boldsymbol{w}_{h}\right)\right\|_{T}^{2}+\left\|\pi_{T}^{0}\left(\widehat{\boldsymbol{u}}_{T}-\boldsymbol{u}_{T}\right)\right\|_{T}\right\} .
$$

Estimating the first term between braces using (50), observing, for the second, that it holds $\left\|\pi_{T}^{0}\left(\widehat{\boldsymbol{u}}_{T}-\boldsymbol{u}_{T}\right)\right\|_{T} \leqslant\left\|\widehat{\boldsymbol{u}}_{T}-\boldsymbol{u}_{T}\right\|_{T}$ since $\pi_{T}^{0}$ is bounded as a projector, and recalling (78), we infer $\left|\mathfrak{T}_{2}\right| \lesssim h^{k+2}\left(\|\boldsymbol{u}\|_{H^{k+2}\left(\mathcal{T}_{h}\right)^{d}}+\|p\|_{H^{k+1}\left(\mathcal{T}_{h}\right)}\right)$. The desired result follows.

\subsection{Numerical example}

We solve the Stokes problem (57) on the unit square $\Omega=(0,1)^{2}$ with $\boldsymbol{f} \equiv \mathbf{0}$ and Dirichlet boundary conditions inferred from the following exact solution:

$$
\boldsymbol{u}(x, y)=(-\exp (x)(y \cos y+\sin y), \exp (x)(y \sin y)), \quad p=2 \exp (x) \sin (y)-p_{0},
$$

where $p_{0} \in \mathbb{R}$ is chosen so as to ensure $\int_{\Omega} p=0$. We consider the three mesh families depicted in Figure 1. The triangular and Cartesian mesh families correspond, respectively, to the mesh families 1 and 2 of the FVCA5 benchmark [30], whereas the (predominantly) hexagonal mesh family was first introduced in [22].

Figure 2 displays convergence results for the different meshes and polynomial degrees up to 3 . Following (69), we display the $\|\cdot\|_{\mathcal{A}}$-norm of the error in the velocity as well as the $L^{2}$-norm of the error both in the velocity and in the pressure. In all the cases, the numerical results match the order estimates predicted by the theory (in some cases, a slight superconvergence is observed for the pressure at the lowest orders).

Local computations are based on the linear algebra facilities provided by the boost uBLAS library [31]. The local linear systems for the computation of the operators $D_{T}^{k}, \mathfrak{C}_{T}^{k}$, and the local contributions to the bilinear form $A$ are solved using the Cholesky factorization available in uBLAS; cf. equations (86), (87), and (90) below. The global system (involving face unknowns only) is solved using SuperLU [17] through the PETSc 3.4 interface [3]. The tests have been run sequentially on a laptop computer powered by an Intel Core i7-3520 CPU clocked at $2.90 \mathrm{GHz}$ and equipped with $8 \mathrm{~Gb}$ of RAM. 


$$
\multimap-k=0 \multimap \square k=1 \multimap-k=2 \multimap * k=3
$$

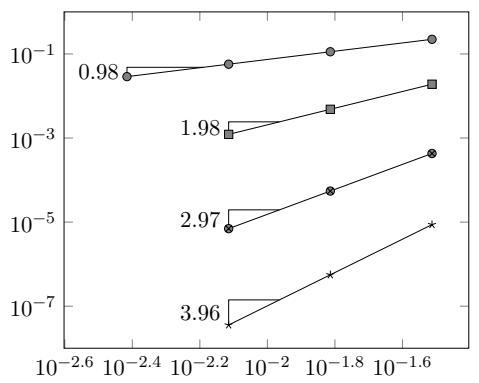

(a) Tria, $\left\|\boldsymbol{w}_{h}-\widehat{\boldsymbol{w}}_{h}\right\|_{\mathcal{A}}$ vs. $h$

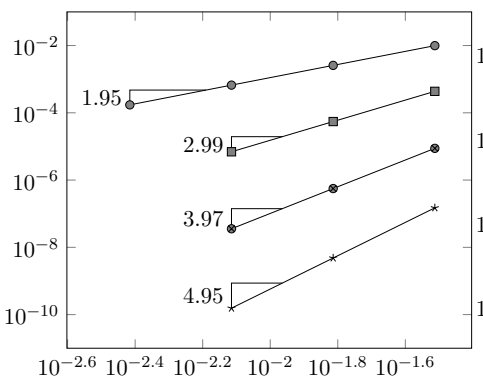

(b) Tria, $\left\|\boldsymbol{u}_{h}-\widehat{\boldsymbol{u}}_{h}\right\|$ vs. $h$

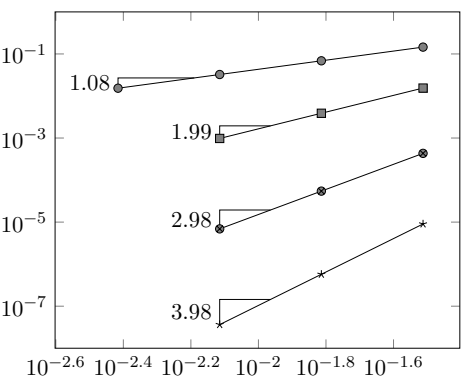

(c) Tria, $\left\|p_{h}-\widehat{p}_{h}\right\|$ vs. $h$

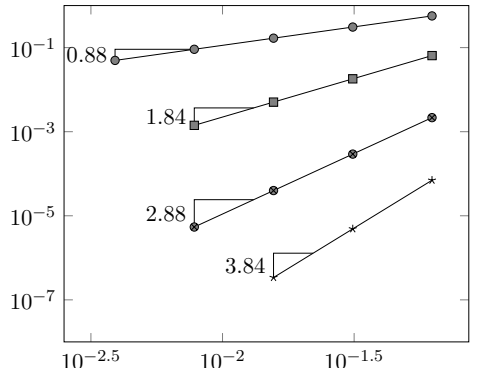

(d) Cart, $\left\|\boldsymbol{w}_{h}-\widehat{\boldsymbol{w}}_{h}\right\|_{\mathcal{A}}$ vs. $h$

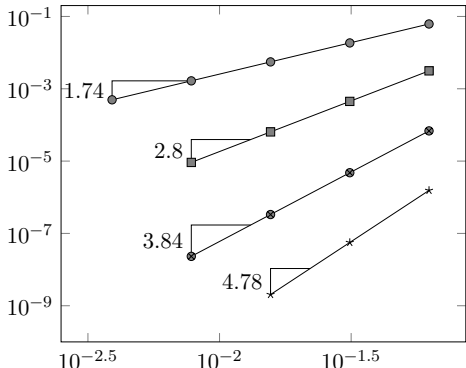

(e) Cart, $\left\|\boldsymbol{u}_{h}-\widehat{\boldsymbol{u}}_{h}\right\|$ vs. $h$

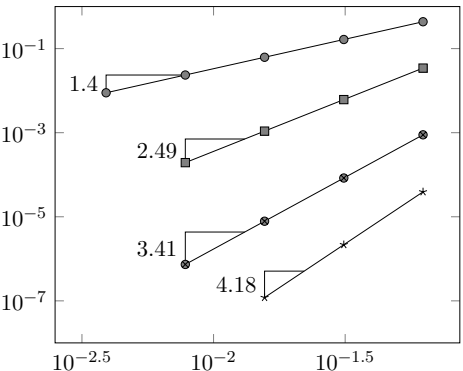

(f) Cart, $\left\|p_{h}-\widehat{p}_{h}\right\|$ vs. $h$

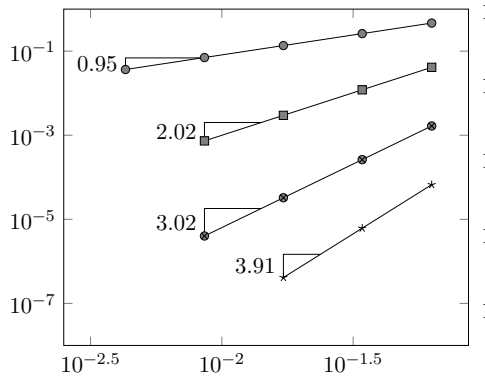

(g) Hex, $\left\|\boldsymbol{w}_{h}-\widehat{\boldsymbol{w}}_{h}\right\|_{\mathcal{A}}$ vs. $h$

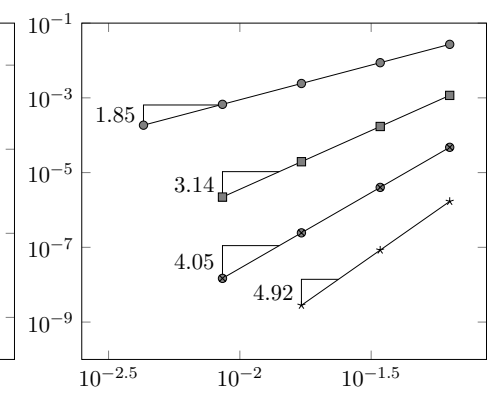

(h) Hex, $\left\|\boldsymbol{u}_{h}-\widehat{\boldsymbol{u}}_{h}\right\|$ vs. $h$

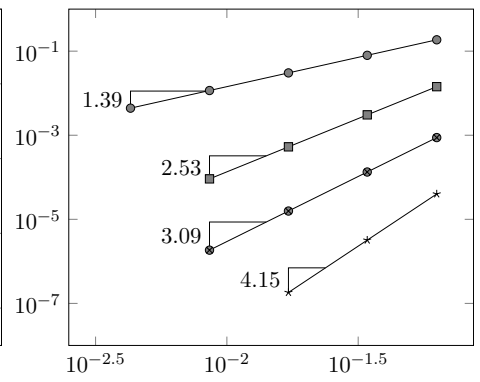

(i) Hex, $\left\|p_{h}-\widehat{p}_{h}\right\|$ vs. $h$

Figure 2: Convergence results for the numerical example of Section 4.6 on the mesh families of Figure 1. The notation is the same as in Theorems 8 and 9 


\section{Implementation}

In this section we discuss the practical implementation of the primal hybrid method (55) for the Poisson problem. The implementation of the method (67) for the Stokes equations follows similar principles and is not detailed here for the sake of brevity.

An essential point consists in selecting appropriate bases for the polynomial spaces on elements and faces. Particular care is required to make sure that the resulting local problems are wellconditioned, since the accuracy of the local computations may affect the overall quality of the approximation. For a given polynomial degree $l \in\{k, k+1\}$, one possibility leading to a hierarchical basis for $\mathbb{P}_{d}^{l}(T), T \in \mathcal{T}_{h}$, is to choose the following family of monomial functions:

$$
\left\{\varphi_{T}=\prod_{i=1}^{d} \xi_{T, i}^{\alpha_{i}} \quad \mid \quad \xi_{T, i}:=\frac{x_{i}-x_{T, i}}{h_{T}} \quad \forall 1 \leqslant i \leqslant d, \quad \underline{\alpha} \in \mathbb{N}^{d}, \quad\|\underline{\alpha}\|_{l^{1}} \leqslant l\right\},
$$

where $\boldsymbol{x}_{T}$ denotes the barycenter of $T$. The idea is here (i) to express basis functions with respect to a reference frame local to one element, which ensures that the basis does not depend on the position of the element and (ii) to scale with respect to a local length scale. Choosing this length scale equal to $h_{T}$ ensures that the basis functions take values in the interval $[-1,1]$. For anisotropic elements, a better option would be to use the inertial frame of reference and, possibly, to perform orthonormalization, cf. [4]. Similarly, a hierarchical monomial basis can be defined for the spaces $\mathbb{P}_{d}^{k}(F), F \in \mathcal{F}_{h}$, using the face barycenter $\boldsymbol{x}_{F}$ and the face diameter $h_{F}$.

Let, for a given polynomial degree $l \geqslant 0$ and a number of variables $n \geqslant 0, N_{n}^{l}:=\operatorname{dim}\left(\mathbb{P}_{n}^{l}\right)$. For any element $T \in \mathcal{T}_{h}$, we assume for the sake of simplicity that a hierarchical basis $\mathcal{B}_{T}^{k+1}:=$ $\left\{\varphi_{T}^{i}\right\}_{0 \leqslant i<N_{d}^{k+1}}$ (not necessarily given by (83)) has been selected for $\mathbb{P}_{d}^{k+1}(T)$ so that $\varphi_{T}^{0}$ is the constant function on $T$ and $\left(\varphi_{T}^{i}, \varphi_{T}^{0}\right)_{T}=0$ for all $1 \leqslant i<N_{d}^{k+1}$. While this latter condition is not verified for general element shapes by the choice (83), one can obtain also in that case a well-posed local problem (21) for the computation of $\mathfrak{C}_{T}^{k}$ by removing $\varphi_{T}^{0}$, since the remaining functions vanish at $\boldsymbol{x}_{T}$. For more general choices, the zero-average condition can be enforced by a Lagrange multiplier constant over the element. Having assumed that $\mathcal{B}_{T}^{k+1}$ is hierarchical, a basis for $\mathbb{P}_{d}^{k}(T)$ is readily obtained by selecting the first $N_{d}^{k}$ basis functions. Additionally, for any face $F \in \mathcal{F}_{h}$, we denote by $\mathcal{B}_{F}^{k}:=\left\{\varphi_{F}^{i}\right\}_{0 \leqslant i<N_{d-1}^{k}}$ a basis for $\mathbb{P}_{d}^{k}(F)$ (not necessarily hierarchical in this case).

The definition of the discrete spaces (10) relies on a generalized notion of DOFs. Solving the primal hybrid problem (55) amounts to computing the coefficients $\left(u_{T}^{i}\right)_{0 \leqslant i<N_{d}^{k}}$ for all $T \in \mathcal{T}_{h}$ and $\left(\lambda_{F}^{i}\right)_{0 \leqslant i<N_{d-1}^{k}}$ for all $F \in \mathcal{F}_{h}$ of the following expansions for the local potential unknown $u_{T} \in U_{T}^{k}$ and the local Lagrange multiplier $\lambda_{F} \in \Lambda_{F}^{k}$, respectively:

$$
u_{T}=\sum_{0 \leqslant i<N_{d}^{k}} u_{T}^{i} \varphi_{T}^{i}, \quad \lambda_{F}=\sum_{0 \leqslant i<N_{d-1}^{k}} \lambda_{F}^{i} \varphi_{F}^{i} .
$$

For all $T \in \mathcal{T}_{h}$, we also introduce as intermediate unknowns the algebraic flux DOFs $\left(\sigma_{T}^{i}\right)_{1 \leqslant i<N_{d}^{k}}$ and $\left(\sigma_{T F}^{i}\right)_{0 \leqslant i<N_{d-1}^{k}}, F \in \mathcal{F}_{T}$, corresponding to the coefficients of the following expansions for 
the components of the local flux unknown $\left(\boldsymbol{\sigma}_{T},\left(\sigma_{T F}\right)_{F \in \mathcal{F}_{T}}\right) \in \boldsymbol{\Sigma}_{T}^{k}$ :

$$
\mathbb{T}_{T}^{k} \ni \boldsymbol{\sigma}_{T}=\sum_{1 \leqslant i<N_{d}^{k}} \sigma_{T}^{i} \boldsymbol{\nabla} \varphi_{T}^{i} \quad \mathbb{F}_{F}^{k} \ni \sigma_{T F}=\sum_{0 \leqslant i<N_{d-1}^{k}} \sigma_{T F}^{i} \varphi_{F}^{i} \quad \forall F \in \mathcal{F}_{T}
$$

where we have used the fact that $\left(\nabla \varphi_{T}^{i}\right)_{1 \leqslant i<N_{d}^{k}}$ is a basis for the DOF space $\mathbb{T}_{T}^{k}$ defined by (9) (the sum starts from 1 to accomodate the zero-average constraint in the definition of $\mathbb{T}_{T}^{k}$ ). Clearly, the total number of local flux DOFs in $\boldsymbol{\Sigma}_{T}^{k}$ (cf. (10)) is

$$
N_{\Sigma, T}^{k}:=\left(N_{d}^{k}-1\right)+\mathfrak{N}_{T} N_{d-1}^{k},
$$

with $\mathfrak{N}_{T}$ defined in (1).

For a given element $T \in \mathcal{T}_{h}$, the discrete operators $D_{T}^{k}, \mathfrak{C}_{T}^{k}, \boldsymbol{\varsigma}_{T}^{k}$ act on and take values in finite dimensional spaces, hence they can be represented by matrices once the choice of the bases for the DOF spaces has been made. Their action on a vector of DOFs then results from right matrix-vector multiplication. In what follows, we show how to carry out the computation of such matrices in detail and how to use them to infer the local contribution to the bilinear form $A$ stemming from the element $T$.

\subsection{Discrete divergence operator}

The discrete divergence operator $D_{T}^{k}$ acting on $\boldsymbol{\Sigma}_{T}^{k}$ with values in $\mathbb{P}_{d}^{k}(T)$ can be represented by the matrix $\mathrm{D}$ of size $N_{d}^{k} \times N_{\Sigma, T}^{k}$ with block-structure $\left[\mathrm{D}_{T}\left(\mathrm{D}_{F}\right)_{F \in \mathcal{F}_{T}}\right]$ induced by the geometric items to which flux DOFs in $\boldsymbol{\Sigma}_{T}^{k}$ are associated. According to the definition (17) of $D_{T}^{k}$, the matrix $\mathrm{D}$ can be computed as the solution of the following linear system of size $N_{d}^{k}$ with $N_{\Sigma, T}^{k}$ right-hand sides:

$$
\mathrm{M}_{D} \mathrm{D}=\mathrm{R}_{D}
$$

with block form
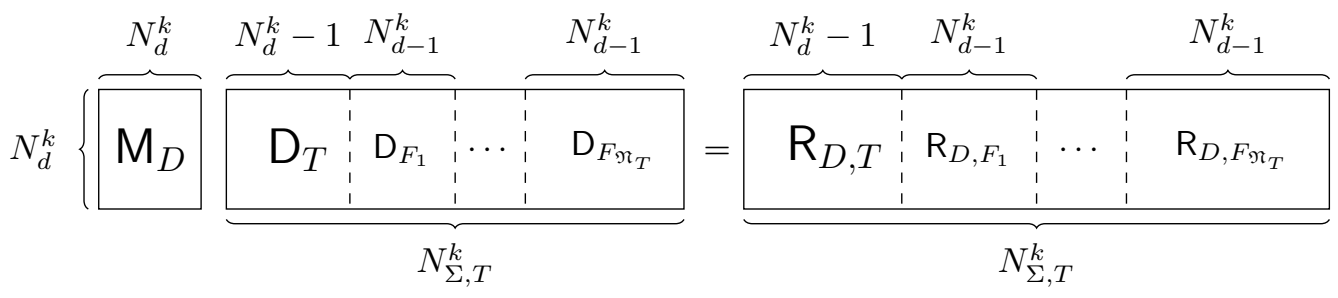

where the system matrix is $\mathrm{M}_{D}:=\left[\left(\varphi_{T}^{i}, \varphi_{T}^{j}\right)_{T}\right]_{0 \leqslant i, j<N_{d}^{k}}$, while the right-hand side is such that

$$
\mathrm{R}_{D, T}:=\left[\left(\nabla \varphi_{T}^{i}, \nabla \varphi_{T}^{j}\right)_{T}\right]_{0 \leqslant i<N_{d}^{k}, 1 \leqslant j<N_{d}^{k}} \quad \mathrm{R}_{D, F}:=\left[\left(\varphi_{T}^{i}, \varphi_{F}^{j}\right)_{F}\right]_{0 \leqslant i<N_{d}^{k}, 0 \leqslant j<N_{d-1}^{k}} \quad \forall F \in \mathcal{F}_{T} .
$$

When considering orthonormal bases such as, e.g., the ones introduced in [4], the matrix $\mathrm{M}_{D}$ is unit diagonal and numerical resolution is unnecessary. 


\subsection{Consistent flux reconstruction operator}

The consistent flux reconstruction operator $\mathfrak{C}_{T}^{k}$ acting on $\boldsymbol{\Sigma}_{T}^{k}$ with values in $\boldsymbol{\nabla} \mathbb{P}_{d}^{k+1,0}(T)$ can be represented by the matrix $C$ of size $\left(N_{d}^{k+1}-1\right) \times N_{\Sigma, T}^{k}$ with the block-structure $\left[\mathrm{C}_{T}\left(\mathrm{C}_{F}\right)_{F \in \mathcal{F}_{T}}\right]$ induced by the geometric items to which flux DOFs in $\boldsymbol{\Sigma}_{T}^{k}$ are associated. According to definition (21a), this requires to solve a linear system of size $\left(N_{d}^{k+1}-1\right)$ with $N_{\Sigma, T}^{k}$ right-hand sides,

$$
\mathrm{M}_{C} \mathrm{C}=\mathrm{Q}_{C} \mathrm{D}+\mathrm{R}_{C}:=\widetilde{\mathrm{R}}_{C} .
$$

The linear system (87) has the following block form:

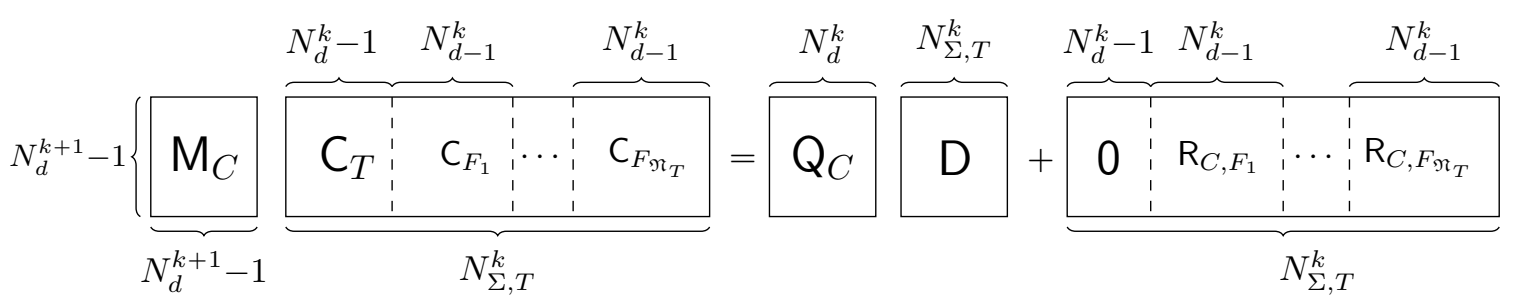

with system matrix $\mathrm{M}_{C}:=\left[\left(\nabla \varphi_{T}^{i}, \nabla \varphi_{T}^{j}\right)\right]_{1 \leqslant i, j<N_{d}^{k+1}}$ and the matrix blocks appearing in the right-hand side in addition to the matrix $\mathrm{D}$ obtained solving (86) are given by

$\mathrm{Q}_{C}:=\left[-\left(\varphi_{T}^{i}, \varphi_{T}^{j}\right)_{T}\right]_{1 \leqslant i<N_{d}^{k+1}, 0 \leqslant j<N_{d}^{k}}, \quad \mathrm{R}_{C, F}:=\left[\left(\varphi_{T}^{i}, \varphi_{F}^{j}\right)_{F}\right]_{1 \leqslant i<N_{d}^{k+1}, 0 \leqslant j<N_{d-1}^{k}} \quad \forall F \in \mathcal{F}_{T}$.

\subsection{Bilinear form $H_{T}$}

We are now ready to compute the matrix $\mathrm{H}$ of size $N_{\Sigma, T}^{k} \times N_{\Sigma, T}^{k}$ representing the local bilinear form $H_{T}$ defined by $(26)$ as

$$
\mathrm{H}=\mathrm{C}^{t} \widetilde{\mathrm{R}}_{C}+\mathrm{J},
$$

where the factors appearing in the first term are defined in (87), while the matrix $\mathrm{J}$ representing the stabilization term $J_{T}$ defined by (28) is given by (the block partitioning is the one induced by the geometric entity to which flux DOFs are attached):

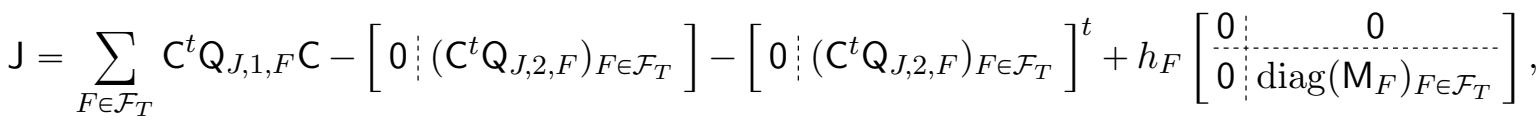

where $C$ is defined by (87) while, for all $F \in \mathcal{F}_{T}$, we have defined the auxiliary matrices

$$
\begin{aligned}
& \mathrm{Q}_{J, 1, F}:=h_{F}\left[\left(\nabla \varphi_{T}^{i} \cdot \boldsymbol{n}_{T F}, \nabla \varphi_{T}^{j} \cdot \boldsymbol{n}_{T F}\right)_{F}\right]_{1 \leqslant i, j<N_{d}^{k+1}}, \\
& \mathrm{Q}_{J, 2, F}:=h_{F}\left[\left(\nabla \varphi_{T}^{i} \cdot \boldsymbol{n}_{T F}, \varphi_{F}^{j}\right)_{F}\right]_{1 \leqslant i<N_{d}^{k+1}, 0 \leqslant j<N_{d-1}^{k}},
\end{aligned}
$$

and face mass matrices

$$
\mathrm{M}_{F}:=\left[\left(\varphi_{F}^{i}, \varphi_{F}^{j}\right)_{F}\right]_{0 \leqslant i, j<N_{d-1}^{k}}
$$




\subsection{Hybridization}

The first step to perform hybridization is to construct the matrix B representing the bilinear form $B$ defined by (33a), which has the following block form corresponding to the geometric items to which DOFs in $\boldsymbol{\Sigma}_{T}^{k}$ (rows) and $W_{T}^{k}$ (columns) are associated:

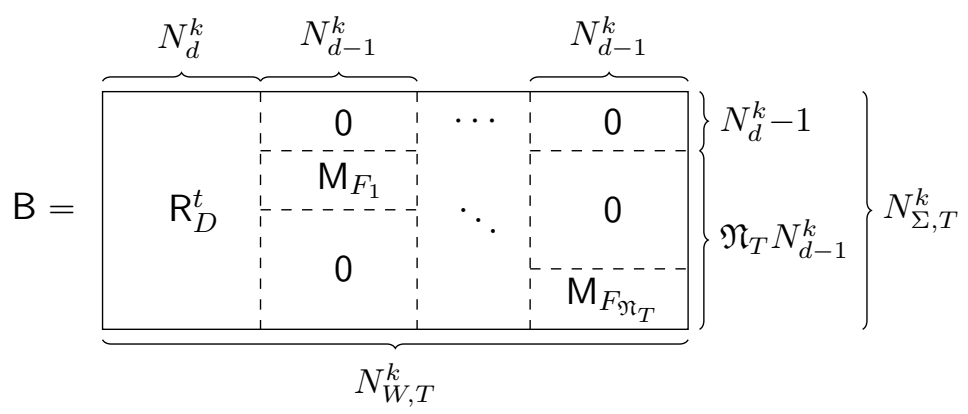

with matrix $\mathrm{R}_{D}$ as in (86), $\mathrm{M}_{F}$ defined by (89), and

$$
N_{W, T}^{k}:=N_{d}^{k}+\mathfrak{N}_{T} N_{d-1}^{k},
$$

corresponding to the number of DOFs in $W_{T}^{k}$.

The condition on the Lagrange multipliers in $\Lambda_{h}^{k}$ on boundary faces $F \in \mathcal{F}_{b}$ (cf. (31)) is enforced via Lagrange multipliers in $\mathbb{P}_{d-1}^{k}(F)$. This choice is reflected by the fact that we include boundary faces in the definition of the matrix $B$.

The local contribution to the bilinear form $A$ defined by (53) is finally given by

$$
A=B^{t} H^{-1} B
$$

which requires the solution of a linear system involving the matrix $\mathrm{H}$ defined by (88). Observe that $\mathrm{H}^{-1} \mathrm{~B}$ is in fact the matrix representation of the lifting operator $\boldsymbol{\varsigma}_{T}^{k}$ defined by (41a).

The matrix $A$ has the following block structure induced by the geometric items to which DOFs in $W_{T}^{k}$ are attached:

$$
\mathrm{A}=\overbrace{\left.\begin{array}{c:c}
\mathrm{A}_{T T} & \mathrm{~A}_{T F} \\
\hdashline \mathrm{A}_{T F}^{t} & \mathrm{~A}_{F F}^{k}
\end{array}\right\} \mathfrak{N}_{T} N_{d-1}^{k}}^{N_{d}^{k}}\} \mathfrak{N}_{T} N_{d-1}^{k}
$$

Observing that cell DOFs for a given element $T$ are only linked to the face DOFs (Lagrange multipliers) attached to the faces in $\mathcal{F}_{T}$, one can finally obtain a problem in the sole Lagrange multipliers by computing the Schur complement of $\mathrm{A}_{T T}$. This requires the numerical inversion of the symmetric positive-definite matrix $\mathrm{A}_{T T}$ of size $N_{d}^{k} \times N_{d}^{k}$ 


\section{References}

[1] C. Amrouche and V. Girault. On the existence and regularity of the solution of Stokes problem in arbitrary dimension. Proc. Japan. Acad., 67:171-175, 1991.

[2] D. N. Arnold and F. Brezzi. Mixed and nonconforming finite element methods: implementation, postprocessing and error estimates. RAIRO Modél. Math. Anal. Num., 19(4):7-32, 1985.

[3] S. Balay, J. Brown, K. Buschelman, W. D. Gropp, D. Kaushik, M. G. Knepley, L. Curfman McInnes, B. F. Smith, and H. Zhang. PETSc Web page. http://www.mcs.anl.gov/petsc, 2011.

[4] F. Bassi, L. Botti, A. Colombo, D. A. Di Pietro, and P. Tesini. On the flexibility of agglomeration based physical space discontinuous Galerkin discretizations. J. Comput. Phys., 231(1):45-65, 2012.

[5] L. Beirão da Veiga, F. Brezzi, A. Cangiani, G. Manzini, L. D. Marini, and A. Russo. Basic principles of virtual element methods. M3AS Math. Models Methods Appl. Sci., 199(23):199-214, 2013.

[6] L. Beirão da Veiga, F. Brezzi, and L. D. Marini. Virtual elements for linear elasticity problems. SIAM J. Numer. Anal., 2(51):794-812, 2013.

[7] L. Beirão da Veiga, F. Brezzi, L.D. Marini, and A. Russo. $H$ (div) and $H$ (curl) conforming VEM, July 2014. Preprint arXiv:1407.6822.

[8] L. Beirão da Veiga, V. Gyrya, K. Lipnikov, and G. Manzini. Mimetic finite difference method for the Stokes problem on polygonal meshes. J. Comput. Phys., 228(19):7215-7232, 2009.

[9] L. Beirão da Veiga, K. Lipnikov, and G. Manzini. Arbitrary-order nodal mimetic discretizations of elliptic problems on general meshes. SIAM J. Numer. Anal., 5(49):1737-1760, 2011.

[10] F. Brezzi and M. Fortin. Mixed and hybrid finite element methods, volume 15 of Springer Series in Computational Mathematics. Springer-Verlag, New York, 1991.

[11] J. Carrero, B. Cockburn, and D. Schötzau. Hybridized globally divergence-free LDG methods. part I: The Stokes problem. Math. Comp., 75:533-563, 2006.

[12] P. Castillo, B. Cockburn, I. Perugia, and D. Schötzau. An a priori error analysis of the local discontinuous Galerkin method for elliptic problems. SIAM J. Numer. Anal., 38(5):1676-1706 (electronic), 2000.

[13] L. Cattabriga. Su un problema al contorno relativo al sistema di equazioni di Stokes. Rend. Sem. Mat. Univ. Padova, 31:308-340, 1961.

[14] B. Cockburn, D. A. Di Pietro, and A. Ern. Bridging the Hybrid High-Order and Hybridizable Discontinuous Galerkin methods. Submitted. Preprint hal-01115318, 2015.

[15] B. Cockburn, J. Gopalakrishnan, and R. Lazarov. Unified hybridization of discontinuous Galerkin, mixed, and continuous Galerkin methods for second order elliptic problems. SIAM J. Numer. Anal., 47(2):1319$1365,2009$.

[16] B. Cockburn, N.C. Nguyen, and J. Peraire. A comparison of HDG methods for Stokes flow. J. Sci. Comput., 45:215-237, 2010.

[17] J. W. Demmel, S. C. Eisenstat, J. R. Gilbert, X. S. Li, and J. W. H. Liu. A supernodal approach to sparse partial pivoting. SIAM J. Matrix Analysis and Applications, 20(3):720-755, 1999.

[18] D. A. Di Pietro and A. Ern. Mathematical aspects of discontinuous Galerkin methods, volume 69 of Mathématiques \& Applications. Springer-Verlag, Berlin, 2012.

[19] D. A. Di Pietro and A. Ern. Arbitrary order mixed methods for heterogeneous anisotropic diffusion on general meshes. Submitted. Preprint hal-00918482, 2013.

[20] D. A. Di Pietro and A. Ern. A hybrid high-order locking-free method for linear elasticity on general meshes. Comput. Meth. Appl. Mech. Engrg., 283:1-21, 2015.

[21] D. A. Di Pietro, A. Ern, and S. Lemaire. An arbitrary-order and compact-stencil discretization of diffusion on general meshes based on local reconstruction operators. Comput. Meth. Appl. Math., 14(4):461-472, 2014.

[22] D. A. Di Pietro and S. Lemaire. An extension of the Crouzeix-Raviart space to general meshes with application to quasi-incompressible linear elasticity and Stokes flow. Math. Comp., 84(291):1-31, 2015.

[23] D. A. Di Pietro and M. Vohralík. A review of recent advances in discretization methods, a posteriori error analysis, and adaptive algorithms for numerical modeling in geosciences. Oil $\&$ Gas Science and Technology, 69(4):701-730, 2014. 
[24] J. Droniou. Finite volume schemes for diffusion equations: introduction to and review of modern methods. Math. Models Methods Appl. Sci., 24:1575-1619, 2014.

[25] T. Dupont and R. Scott. Polynomial approximation of functions in Sobolev spaces. Math. Comp., 34(150):441-463, 1980.

[26] H. Egger and J. Schöberl. A mixed-hybrid-discontinuous galerkin finite element method for convectiondiffusion problems. IMA Journal of Numerical Analysis, 30(4):1206-1234, 2010.

[27] H. Egger and C. Waluga. hp-analysis of a Hybrid DG method for Stokes flow. IMA Journal of Numerical Analysis, 33(2):687-721, 2013.

[28] A. Ern and J.-L. Guermond. Theory and Practice of Finite Elements, volume 159 of Applied Mathematical Sciences. Springer-Verlag, New York, NY, 2004.

[29] V. Girault, B. Rivière, and M. F. Wheeler. A discontinuous Galerkin method with nonoverlapping domain decomposition for the Stokes and Navier-Stokes problems. Math. Comp., 74(249):53-84, 2004.

[30] R. Herbin and F. Hubert. Benchmark on discretization schemes for anisotropic diffusion problems on general grids. In R. Eymard and J.-M. Hérard, editors, Finite Volumes for Complex Applications V, pages 659-692. John Wiley \& Sons, 2008.

[31] W. Joerg and M. Koch. BOOST uBLAS C++ Library. http://www.boost.org.

[32] R.J. Labeur and G.N. Wells. Energy stable and momentum conserving hybrid fininte element method for the incompressible Navier-Stokes equations. SIAM J. Sci. Comput., 34(2):A889-A913, 2012.

[33] C. Lehrenfeld. Hybrid Discontinuous Galerkin methods for solving incompressible flow problems. PhD thesis, Rheinisch-Westfälischen Technischen Hochschule Aachen, 2010.

[34] K. Lipnikov and G. Manzini. A high-order mimetic method on unstructured polyhedral meshes for the diffusion equation. J. Comput. Phys., 272:360-385, 2014.

[35] N. C. Nguyen, J. Peraire, and B. Cockburn. A hybridizable discontinuous Galerkin method for Stokes flow. Comput. Methods Appl. Mech. Engrg., 199:582-597, 2010.

[36] A. Toselli. $h p$-finite element discontinuous Galerkin approximations for the Stokes problem. M3AS, 12(11):1565-1616, 2002.

[37] M. Vohralík and B. Wohlmuth. Mixed finite element methods: implementation with one unknown per element, local flux expressions, positivity, polygonal meshes, and relations to other methods. M3AS Math. Models Methods Appl. Sci., 23(5):803-838, 2013. 